\title{
Comment mener un exercice de couverture : Outil d'évaluation rapide de programmes et services
}

\author{
Carey Meyers \\ Population Council \\ Solene Lardoux
}

Follow this and additional works at: https://knowledgecommons.popcouncil.org/departments_sbsr-pgy

Part of the Demography, Population, and Ecology Commons, Family, Life Course, and Society Commons, International Public Health Commons, and the Quantitative, Qualitative, Comparative, and Historical Methodologies Commons How does access to this work benefit you? Let us know!

\section{Recommended Citation}

Meyers, Carey and Solene Lardoux. 2006. "Comment mener un exercice de couverture : Outil d'évaluation rapide de programmes et services." New York: Population Council. 


\section{Comment mener un exercice de couverture : \\ Outil d'évaluation rapide de programmes et services}

* Comment mener un exercice de couverture est disponible en anglais, portugais et espagnol.

\section{(1) Population Counal \\ Juillet 2006}

Copyright @ 2006 The Population Council, Inc. 


\section{(2) Population Council}

\section{$\underline{\text { Remerciements }}$}

Le concept original de l'exercice de couverture $(E C)$ a été présenté à l'occasion d'un atelier du Population Council et du Fonds des Nations Unies pour la population (UNFPA) tenu à New York du $1^{\text {er }}$ au 3 mai 2002. Le rapport de la rencontre a été publié sous le titre de "Transitions to Adulthood: Adolescent and Youth Sexual and Reproductive Health: Charting directions for a second generation of programming » (2003). La notion d'exercice de couverture est définie par Judith Bruce au chapitre 2 du rapport, " Steps in Building Evidence-Based Programs for Adolescents » (accessible en ligne, en anglais, à l'adresse http://www.popcouncil.org/pdfs/AYSRH/2jb1.pdf).

Le premier exercice de couverture a été réalisé en Éthiopie, en 2003, sous les auspices du Population Council (Annabel Erulkar et Tekle-Ab Mekbib) en collaboration avec l'organisation Private Associations Cooperating Together (PACT, sous subvention de l'USAID) et 13 organisations pour les jeunes. Les données ont été présentées dans le cadre de séminaires avec les organisations partenaires en 2004, avant d'être publiées en 2005 (Mekbib, Erulkar et Belete, 2005).

Un exercice de couverture a été entrepris au Burkina Faso dans le contexte d'un partenariat entre l'UNFPA, le Population Council et 20 associations. Geneviève Ah Sue et Siaka Traoré, de I'UNFPA, ont participé au processus, avec l'aide de Zio Simplice Batebié, au titre de consultant.

Des exercices de couverture ont également été menés en Mauritanie et en Guinée-Bissau, dans le cadre d'une collaboration entre le Population Council, I'UNFPA et les organisations locales. Mohamed Baraket, Seynath Aidara, Elhacen Mohamed Abdellahi, Delphine Vazeilles et Yao Gaspard Bossou de I'UNFPA, Bih Abdel Kader et Moctar Ould Ndiack, de la Direction de la Jeunesse, et Mohamed Aly Ould Ekeibed, au titre de consultant ont participé à l'exercice de Mauritanie. En Guinée-Bissau, les participants sont Guy de Araujo, Joaquim Gomes, Candida Lopes et Nélida Rodrigues de I'UNFPA, N'kitcha No Obna et Mauricio Sanca, de I'Institut de la Jeunesse, et Placido Cardoso, consultant.

La préparation du présent guide par le Population Council est le produit d'une collaboration avec Futures Group, en vertu du contrat USAID n HRN-C-00-00-00006-00, et avec l'assistance complémentaire du Department for International Development (DfID) (à travers le programme pour adolescentes du Population Council) et de l'UNFPA. Les auteurs de ce guide, Carey Meyers et Solène Lardoux, tiennent à remercier Annabel Erulkar, Heidi Jones, Amy Joyce, Judith Bruce, Saumya RamaRao et leurs collaborateurs au Futures Group pour leurs précieuses contributions de fond et à la rédaction.

Toute partie de ce guide peut être copiée ou adaptée, si besoin est, sans en demander la permission au Population Council, à condition que les parties copiées soient distribuées gratuitement ou sans profit, et que la source soit identifiée par citation. Toute reproduction commerciale nécessite d'obtenir une permission préalable du Population Council. Le Population Council apprécierait de recevoir une copie de tout matériel dans lequel le texte est utilisé, ou de tout rapport dont les résultats sont issus de l'utilisation de la technique de l'exercice de couverture. Correspondance à envoyer à :

Judith Bruce

Population Council

One Dag Hammarskjold Plaza

New York, NY 10017 USA

+1-212-339-0500 (téléphone)

ibruce@popcouncil.org

http://www.popcouncil.org 


\section{Table des matières}

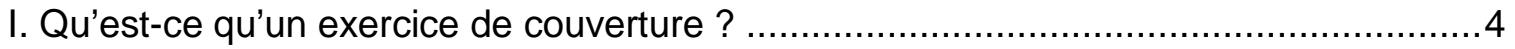

II. Intérêt de l'exercice de couverture ...................................................................... 4

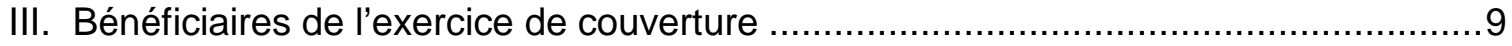

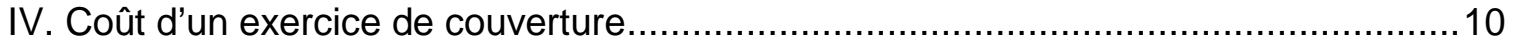

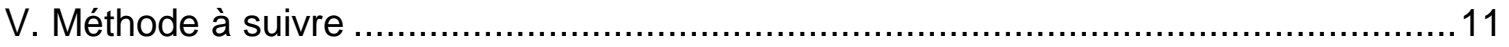

Phase 1. Sélection des partenaires et définition d'un format et d'un cadre communs.... 12

Phase 2. Sensibilisation du personnel et planification générale ...................................14

Finalisation de l'outil de collecte des données (registre d'activité)..................17

Préparation à l'atelier de formation..................................................

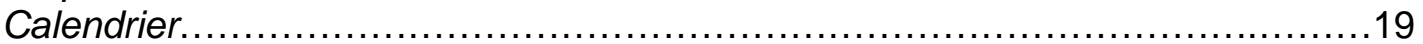

Phase 3. Formation et lancement de la collecte de données.....................................20

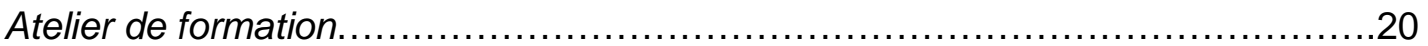

Assistance des responsables adjoints aux pairs éducateurs ou autres

prestataires..........................................................................

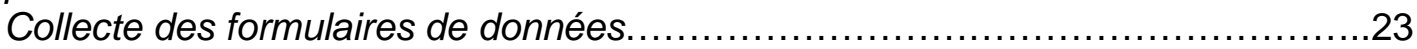

Configuration des écrans de saisie des données................................23

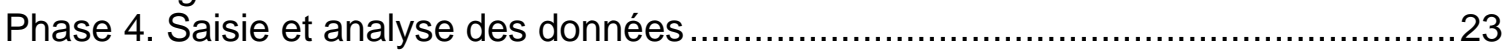

Saisie et analyse des données................................................. 24

Présentation des résultats préliminaires pour dissémination......................26

Phase 5. Dissémination des données et réévaluation des programmes et politiques en fonction de l'éducation par les pairs.................................................................. 27

Atelier de dissémination à l'intention des pairs éducateurs, prestataires et responsables de programme...................................................... 27

Atelier de dissémination à l'intention des donateurs, organismes gouvernementaux, décideurs politiques et autres parties prenantes...................................27

Révision des programmes pour mieux saisir les destinataires insuffisamment touchés par les modèles existants - ou détermination d'autres méthodes d'accès à

ceux et celles qui ne participent pas aux programmes ............................28

Annexe 1.1 : Formulaire de collecte de données pour tabulation manuelle ..................29

Annexe A.2 : Feuille de calcul : Mappage de contenu des programmes pour

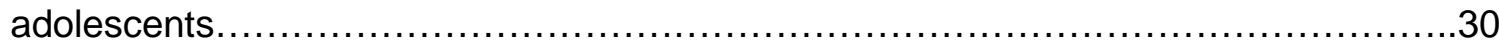

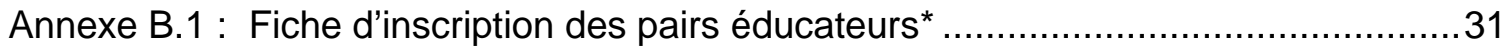

Annexe B.2 : Formulaire de collecte de données destiné aux organisations ................32

Annexe B.3 : Registre d'activité individuelle pour pair éducateur ou prestataire ............33

Annexe B.4 : Fiche d'inscription pour activités de groupe des pairs éducateurs ${ }^{\star}$...........34

Annexe B.5 : Registre d'activité de centre de jeunesse ............................................ 35

Annexe C : Ordre du jour de la formation au Burkina Faso.......................................36

Annexe D : Exemples de résultats de l'exercice de couverture réalisé en Mauritanie...37 


\section{Qu'est-ce qu'un exercice de couverture ?}

Un exercice de couverture (EC) est un instrument d'évaluation simple, économique et rapide pouvant servir à profiler les personnes touchées par un prestataire donné, un groupe de prestataires ${ }^{1}$ ou des organisations partageant une clientèle commune dans une zone géographique particulière. Cet instrument a été mis au point pour assister les programmes de jeunesse, mais il peut également être utilisé par d'autres bénéficiaires ayant des services offerts en établissement ou en antenne. L'EC collecte les données relatives à différentes caractéristiques, y compris le sexe, l'état de scolarisation, l'habitat, l'activité rémunératrice et l'état matrimonial des personnes bénéficiant d'un programme ou d'un service. II permet au personnel et aux responsables des programmes d'évaluer systématiquement les services qu'ils fournissent, l'endroit exact de leur prestation et les caractéristiques de leurs bénéficiaires (clients habituels ou non). Le but ultime de l'instrument est de déterminer : (1) si les services atteignent leurs bénéficiaires prévus et (2) s'ils sont appropriés et conviennent aux personnes qui les reçoivent.

L'EC apporte aux organisations communautaires d'inspiration religieuse et non gouvernementales, aux programmes locaux de l'état, ainsi qu'aux partenaires de mise en œuvre et aux organismes de coopération associés à USAID, un instrument élémentaire convivial et rentable de suivi et d'évaluation ( $S+E)$. Comprendre si les programmes touchent ou non les bénéficiaires auxquels ils s'adressent aide les responsables de leur mise en œuvre comme les donateurs à concentrer leurs activités et leurs ressources de manière à atteindre ceux qui en ont le plus besoin.

\section{Intérêt de l'exercice de couverture}

Les chercheurs du Population Council ont mis au point l'EC dans le but d'offrir aux organisations pour les jeunes un instrument de suivi et d'évaluation qui reconnaisse la diversité des populations adolescentes et de leur vécu. Parfois omise, la reconnaissance des besoins distincts des adolescents d'âge, sexe, état matrimonial et autres caractéristiques différents, est pourtant essentielle à la compréhension des vulnérabilités de l'adolescence (voir l'encadré ci-dessous pour plus de détails). Une fille célibataire de 12 ans, non scolarisée, vivant loin de son foyer rural avec des « cousins » éloignés qui

\footnotetext{
${ }^{1}$ Le terme «prestataire » désigne toute personne assurant la prestation de « services » tels que l'échange d'information dans les programmes d'éducation par les pairs ou par le personnel d'un centre de jeunesse. Le terme ne se limite pas aux travailleurs de la santé.
} 
l'emploient comme domestique dans un milieu urbain peu familier a des besoins nettement distincts de ceux d'une jeune fille de 19 ans instruite, fiancée et vivant encore avec ses deux parents. Les deux ont également des besoins bien différents de ceux d'un jeune homme de 16 ans vivant en milieu semi-rural, orphelin du sida et s'efforçant de poursuivre ses études tout en subvenant aux besoins de ses petits frères et sœurs. Les données nationales, recueillies dans le cadre des Enquêtes démographiques et de santé (EDS) par exemple, peuvent être utiles à la description des proportions d'adolescents d'un pays tombant dans ces différents types de catégories. L'encadré cidessous décrit en détails, sur la base de données d'EDS, les caractéristiques de différenciation des adolescents. Dans chacune des catégories définies, l'EC met en lumière les adolescents (ou autres populations à l'étude) qui sont effectivement touchés par les programmes conçus à leur intention, et ceux qui ne le sont pas. 
Diversité de la population adolescente : Tabulation par le Population Council des données d'EDS sur les adolescents

Les tableaux « Facts About Adolescents from the Demographic and Health Survey -Données sur les adolescents tirées de l'Enquête démographique et de santé » présentent l'information relative aux jeunes de 10 à 24 ans selon les critères suivants :

- Résidence (urbaine/rurale)

- Distribution démographique

- Habitat

- État de survie des parents et résidence au foyer

- Caractéristiques du chef de ménage

- Inscription scolaire et niveau atteint (milieu urbain/rural)

- État matrimonial

- État matrimonial actuel

- Mariage avant 15, 18 et 20 ans

- Scolarisation et activité rémunératrice des adolescents, en fonction de leur état matrimonial et paternité/maternité

- Activité sexuelle et paternité/maternité

- Connaissances, comportement et risques spéciaux en matière de santé reproductive

- Sensibilisation aux méthodes de contraception modernes et usage de ces méthodes

- Sensibilisation au VIH/sida

- Expérience des infections sexuellement transmissibles

- Excision des filles (données recueillies dans certains pays seulement)

Une seconde série de tableaux a été compilée concernant les très jeunes adolescents (de 10 à 14 ans), en réponse à l'intérêt croissant manifesté à l'égard de ce sous-groupe.

"Selected DHS Data on 10-14-year-olds » couvre beaucoup des dimensions énoncées cidessus (résidence, habitat, scolarisation) et se concentre en outre sur les points suivants :

- Mappage des filles et des garçons en fonction de leur habitat et de leur inscription à l'école, afin de déterminer les proportions dénuées des deux structures de protection que constituent les parents et l'école.

- Relation entre l'habitat et l'inscription à l'école, et différences en fonction du sexe.

- Proportion de filles ayant eu des rapports sexuels, mariées ou ayant accouché avant l'âge de 15 ans.

- Exposition à la radio et à la télévision (milieu urbain/rural).

Voir http://www.popcouncil.org/gfd/gfddhs.html pour plus de détails.

Motivé par les évaluations antérieures sur la portée d'organisations de jeunesse, le personnel du Population Council a mis au point cet outil et l'a appliqué aux programmes pour les jeunes d'Éthiopie (2004), du Burkina Faso (2005), de Mauritanie (2005) et de Guinée-Bissau (2006). L'EC permet aux prestataires et aux organisateurs de services de voir la diversité interne des jeunes qu'ils servent et d'en identifier les proportions par sous-groupes : âge, sexe, scolarisation, état matrimonial, habitat (et autres catégories pertinentes). Les deux tableaux ci-dessous peuvent servir de base aux organisations 
chargées de la collecte de données dans le cadre d'un EC. Ces données peuvent être utiles au commentaire de la question de savoir si leurs programmes touchent ou non, au moment de l'étude, les populations qu'ils visent. Elles peuvent du reste servir à profiler les bénéficiaires effectivement atteints par les programmes, par rapport aux destinataires visés.

Tableau 1. Répartition des adolescents en fonction de leur âge et sexe et de leur état de scolarisation.

\begin{tabular}{|l|c|c|c|}
\hline \multirow{2}{*}{ Âge et sexe } & \multicolumn{3}{l}{ Scolarisation } \\
\cline { 2 - 4 } & $\begin{array}{l}\text { Enseignement } \\
\text { primaire }\end{array}$ & $\begin{array}{l}\text { Enseignement } \\
\text { secondaire }\end{array}$ & Non scolarisé/e/s \\
\hline Filles, 10 à 14 ans & $\mathrm{A}$ & $\mathrm{B}$ & $\mathrm{C}$ \\
\hline Filles, 15 à 19 ans & $\mathrm{D}$ & $\mathrm{E}$ & $\mathrm{F}$ \\
\hline Garçons, 10 à 14 ans & $\mathrm{G}$ & $\mathrm{H}$ & $\mathrm{I}$ \\
\hline Garçons, 15 à 19 ans & $\mathrm{J}$ & $\mathrm{K}$ & $\mathrm{L}$ \\
\hline
\end{tabular}

Tableau 2. Répartition des adolescents en fonction de leur âge et sexe et de leur état matrimonial et habitat

\begin{tabular}{|l|c|c|c|c|}
\hline & \multicolumn{2}{|l|}{ État matrimonial } & \multicolumn{2}{l|}{ Habitat } \\
\cline { 2 - 5 } Âge et sexe & $\begin{array}{l}\text { Marié/e/s avec } \\
\text { ou sans } \\
\text { enfants }\end{array}$ & $\begin{array}{l}\text { Célibataires } \\
\text { avec ou sans } \\
\text { enfants }\end{array}$ & $\begin{array}{l}\text { Deux parents } \\
\text { sous le même } \\
\text { toit }\end{array}$ & $\begin{array}{l}\text { Un seul ou } \\
\text { aucun parent }\end{array}$ \\
\hline $\begin{array}{l}\text { Filles, 10 à 14 } \\
\text { ans }\end{array}$ & $\mathrm{A}$ & $\mathrm{B}$ & $\mathrm{A}$ & $\mathrm{B}$ \\
\hline $\begin{array}{l}\text { Filles, } 15 \text { à 19 } \\
\text { ans }\end{array}$ & $\mathrm{C}$ & $\mathrm{D}$ & $\mathrm{C}$ & $\mathrm{D}$ \\
\hline $\begin{array}{l}\text { Garçons, } 10 \text { à } \\
\mathbf{1 4} \text { ans }\end{array}$ & $\mathrm{E}$ & $\mathrm{F}$ & $\mathrm{E}$ & $\mathrm{F}$ \\
\hline $\begin{array}{l}\text { Garçons, } 15 \text { à } \\
\text { 19 ans }\end{array}$ & $\mathrm{G}$ & $\mathrm{H}$ & $\mathrm{G}$ & $\mathrm{H}$ \\
\hline
\end{tabular}

Source : Adaptation du tableau (p. 41) de " Steps in building evidence-based programs for adolescents » (Judith Bruce), chapitre 2 de Charting Directions for a Second Generation of Programming, document de base à l'atelier de l'UNFPA et du Population Council sur la santé sexuelle et reproductive des adolescents et des jeunes, pour la préparation d'une seconde génération de programmes pour adolescents (Adolescent and Youth Sexual and Reproductive Health: Charting Directions for a Second Generation of Adolescent Programming, New York, 13 mai 2002). 


\section{(1) Population Council}

Dotées des données ainsi produites par un EC, les organisations peuvent décider si leurs programmes atteignent leurs objectifs ou s'il convient de revoir leur approche au service d'un groupe distinct d'adolescents ou d'offrir des services différents à ceux qui en bénéficient déjà.

Dès sa mise en œuvre dans une organisation, l'exercice de couverture apporte une évaluation transversale rapide, tout en établissant une référence pour l'avenir. Caractéristique particulièrement notable des EC, les données sont recueillies par les personnes au contact direct des bénéficiaires du programme. Dans le cadre d'un programme de pairs éducateurs, par exemple, ces derniers effectuent eux-mêmes la collecte. Mieux encore, les données sont obtenues à faible coût, dans le cadre ordinaire des activités du programme. Le matériel nécessaire se limite au formulaire de collecte des données, également appelé registre d'activité (voir les annexes A.1, A.2, B.1, B.3, B.4 et B.5), à un stylo et à un support d'écriture. Un logiciel d'analyse statistique peut accélérer le processus après la collecte, mais les données peuvent tout aussi bien être tabulées manuellement (voir l'annexe A.1).

\footnotetext{
2 II convient, à un certain point, d'entreprendre un exercice parallèle à celui de couverture pour dresser l'inventaire du contenu des programmes proposés par l'état et par les organisations non gouvernementales. L'annexe A.2 présente un exemple du type d'instrument utile à la détermination des " points d'entrée » programmatiques (santé, éducation, vie active, etc.) recevant la plus grosse partie de l'attention et des ressources. Largement indicatif, cet instrument pourra servir de guide très général à l'identification des thèmes ou des sous-groupes de jeunes négligés.
} 


\section{Avantages d'un exercice de couverture}

- Identification par les directeurs de programme et prestataires de services (pairs éducateurs ou personnel de centre de jeunesse) des bénéficiaires touchés ou non.

- Instrument de suivi et d'évaluation.

- Renforcement de capacité ultérieure des programmes de suivi et d'évaluation.

- Facilité d'emploi (n'exige ni haut niveau d'alphabétisme, ni hautes qualifications d'analyse de données).

- Faible technicité et réplicabilité.

- Faible coût.

- Propriété des données.

- Collecte des données effectuée par les prestataires habituels, dans le cadre de leurs activités ordinaires.

- Mécanisme de rétroaction pour les agents qui en disposent rarement.

\section{Bénéficiaires de l'exercice de couverture}

Beaucoup d'intéressés peuvent bénéficier de l'exercice de couverture. Notamment :

- les organisations prestataires individuelles

- un groupe ou une communauté d'organisations au service d'une même clientèle

- un groupe ou une communauté d'organisations opérant dans une zone d'influence donnée (ville, région ou pays)

- les planificateurs de programme

- les donateurs

Les organisations individuelles qui désirent mieux cerner les bénéficiaires effectivement touchés par leur programme ont intérêt à réaliser un EC. Pour les services de jeunes, il peut s'agir de programmes de pairs éducateurs, de programmes axés sur une activité particulière telle que le football ou les compétences de vie, ou encore d'organisations d'inspiration religieuse.

Une communauté d'organisations au service d'une même clientèle, soucieuse d'identifier les personnes touchées par leurs programmes et celles laissées pour 
compte, aura également intérêt à effectuer un EC. Une organisation particulière touchet-elle plus de filles que de garçons ? Quelles sont les stratégies qui permettent à cette organisation d'atteindre davantage les jeunes alors que d'autres en touchent moins? Les problèmes communs peuvent être identifiés par discussions et échanges intergroupes.

Une communauté d'organisations opérant dans une zone d'influence donnée et désireuse de mieux comprendre les différences d'accès à ses services dans la zone considérée trouvera également l'EC utile. Quelles sont les populations atteintes dans la zone ? Certains groupes ne sont-ils pas atteints du tout ? Y a-t-il une différence de profil pour les services reçus en fonction de l'âge, du sexe et des caractéristiques sociodémographiques?

Aux planificateurs de programmes, l'EC permettra d'identifier les lacunes des programmes, ou d'accroître la portée de ceux qui atteignent leurs buts.

Les donateurs, enfin, trouveront dans l'EC un instrument utile au suivi des fonds consentis, ainsi qu'à l'identification des bénéficiaires de l'investissement et des types de services et efforts fructueux. Les résultats d'un EC aident les donateurs à identifier les besoins et à cibler leurs investissements futurs.

\section{Coût d'un exercice de couverture}

Les coûts principaux d'un premier exercice de couverture sont ceux des ateliers de formation (y compris les frais de déplacement et indemnités journalières des participants), de la création et de la photocopie des outils de collecte des données, des ressources humaines nécessaires à la gestion et à l'analyse des données, et des ateliers de dissémination.

L'expérience des exercices menés dans quatre pays d'Afrique peut servir de référence à l'estimation des coûts. Compte tenu d'une participation de 13 à 20 organisations, les coûts locaux directs sont inférieurs à USD 10 000. En Éthiopie, six semaines de collecte de données par 13 grandes organisations de jeunes ont coûté USD 7 000. Au Burkina Faso, la collecte des données par 20 organisations sur une période de six semaines en milieu urbain et rural a coûté moins de USD 15 000. Les frais de collaboration technique 


\section{(1) Population Council}

extérieure aux quatre sites atteignent à ce jour entre USD 10000 et 15000 par site.

Ainsi, tous frais directs inclus, un exercice de couverture impliquant 13 à 20 organisations coûte généralement moins de USD 25000.

Si une organisation communautaire devait réaliser elle-même un exercice de couverture, sans assistance à la recherche et sans partager ses résultats, les coûts seraient limités au prix des instruments de collecte des données et au temps du personnel consacré à la tabulation $^{3}$.

\section{Méthode à suivre}

Un exercice de couverture s'effectue en cinq grandes phases, sur une période d'environ sept mois :

1. Sélection des partenaires et définition d'un format et d'un cadre communs (4 semaines)

2. Sensibilisation du personnel et planification générale (4 semaines)

3. Formation et collecte des données (4 à 6 semaines)

4. Saisie et analyse des données (4 à 6 semaines)

5. Dissémination des données de séminaire et dialogue initial avec les organisations participantes, donateurs et décideurs programmatiques et politiques (1 à 2 jours pour la dissémination)

Que l'exercice soit réalisé en un emplacement centralisé (centres de jeunesse) ou en antenne (programme d'éducation par les pairs), les mêmes phases sont applicables. N'oubliez pas qu'il est possible, et souvent désirable même, de réaliser en même temps un exercice de couverture de programme de pairs éducateurs et d'un centre de jeunesse. Ainsi, lorsqu'un centre de jeunesse propose différentes activités, y compris d'éducation par les pairs, il est possible d'incorporer un exercice de couverture des pairs éducateurs dans une étude plus large du centre de jeunesse. Tandis qu'une équipe se concentre sur l'usage global du programme au centre de jeunesse, les pairs éducateurs peuvent recueillir des données sur les activités d'antenne. Cette double observation

\footnotetext{
${ }^{3}$ L'outil de collecte original (voir l'annexe A.1) a été publié pour la première fois dans «Steps in Building Evidence-Based Programs for Adolescents » (Bruce, 2003). II s'agit du format le plus simple qu'une organisation puisse utiliser, parfaitement adapté aux tabulations manuelles.
} 
permet de bien cerner la portée des services offerts dans un centre ou par un programme de jeunesse.

\section{Phase 1. Sélection des partenaires et définition d'un format et d'un cadre communs}

- Durée estimée : 4 semaines

- Principales activités accomplies:

- Identification des organisations de mise en œuvre

- Collecte d'information de base sur les programmes/organisations à l'étude

L'élément clé de cette étape est la souplesse. Les partenaires varieront suivant les besoins politiques des donateurs ou autres d'un pays ou d'une région. Dans certains endroits, il existe déjà un consortium d'organisations de jeunesse pouvant servir de base. En Éthiopie, par exemple, le groupe PACT d'encadrement technique au soutien d'un réseau d'ONG a collaboré avec le Population Council et le ministère de la Jeunesse. Au Burkina Faso, en Mauritanie et en Guinée Bissau, le personnel de I'UNFPA et du Population Council a identifié les prestataires de services pour les jeunes. Une large proportion des organisations contactées ont exprimé leur désir de participer à la collecte d'information. Dans certains contextes, les donateurs peuvent avoir un intérêt tout particulier dans ces évaluations : elles les aident en effet à prendre des décisions factuelles quant au ciblage de leurs ressources vers les jeunes qui en ont le plus besoin.

Lors de la sélection de leurs partenaires et principaux collaborateurs, les parties prenantes doivent préciser leurs objectifs. Ceux énoncés ci-dessous peuvent être sélectionnés individuellement ou globalement, suivant que les parties prenantes cherchent à identifier :

- les bénéficiaires effectifs d'une organisation ou d'un programme ;

- les sous-groupes atteints par une communauté d'organisations au service d'une même clientèle ;

- les bénéficiaires, au sein d'une zone géographique particulière et pour une population donnée (les adolescents de 10 à 14 ans, par exemple), touchés par tous les programmes ;

- les bénéficiaires touchés par les programmes financés par un donateur particulier. 


\section{(1) Population Council}

Un EC peut servir à profiler les bénéficiaires pour un programme particulier, un groupe de programmes au sein d'une même organisation, une zone d'influence donnée, sur toute la gamme des organisations de jeunesse, au sein d'une région, ou projeter la couverture nationale. En Mauritanie, au Burkina Faso et en Guinée-Bissau, l'UNFPA entendait obtenir des données de couverture représentatives à l'échelle nationale. L'option de couverture nationale implique un accès à grande échelle à plusieurs organismes pour les jeunes et des résultats aptes à contribuer aux plus vastes décisions de politique ainsi qu'aux adaptations programmatiques. Une autre option consiste à concentrer un EC sur une organisation seulement. Par exemple, si un organisme coopérateur envisageait un partenariat avec le plus vaste programme de pairs éducateurs de Yaoundé en vue de l'apport d'une information sanitaire aux filles de 15 à 19 ans non scolarisées des milieux urbains, il pourrait avoir recours à l'EC pour déterminer le succès des prestations existantes.

Pour la collecte d'information sur les populations touchées par plus d'une organisation, il importe que l'équipe chargée de l'EC identifie d'abord les principales organisations intéressées et en sélectionne ensuite un groupe représentatif. Les organisations participant à un EC multisite peuvent toujours apporter leurs données brutes à un effort plus vaste tout en préservant leur anonymat : l'utilité des analyses ne passe pas par l'identification obligatoire d'un programme spécifique.

Une fois identifié/s le ou les programmes participants, il faut décider du responsable de la collecte des données. Une option consiste à faire participer tous les pairs éducateurs ou autres prestataires du ou des programmes considérés. Une autre option serait de prendre un échantillon représentatif des prestataires participants - en faisant particulièrement attention aux caractéristiques d'âge, de sexe et de scolarisation. Si un programme compte 10 pairs éducateurs, dont cinq garçons et cinq filles, on pourrait par exemple confier la tâche à deux garçons et deux filles. Si le programme comptait huit filles et deux garçons, il vaudrait probablement mieux retenir quatre filles et un garçon. Les pairs éducateurs ou autres prestataires les plus actifs peuvent être sélectionnés en raison de leur dévouement au programme. L'idée n'est pas mauvaise, pour autant que ce critère soit pris en compte lors de l'interprétation des résultats. L'une des premières étapes de cette phase consiste donc à recueillir une information pertinente aux 
caractéristiques des pairs éducateurs ou autres prestataires eux-mêmes (voir le formulaire type proposé à l'annexe B.1). Les responsables de la collecte des données identifiés, il faut penser au calendrier et décider d'une période appropriée (entre 1 et 12 semaines) pour que les données représentent adéquatement une année entière.

\section{Phase 2. Sensibilisation du personnel et planification générale}

- Durée estimée : 4 semaines

- Principales activités accomplies:

- engagement des organisations participantes

- sélection de l'équipe

- finalisation de l'outil de collecte des données

- préparation à l'atelier de formation

- élaboration du calendrier de l'EC

La première étape de la phase 2 consiste à identifier la personne responsable de la gestion générale de l'EC (le « coordinateur du projet »), ainsi que le responsable au niveau de chaque organisation ou programme participant. L'établissement d'un terrain d'entente et la bonne compréhension des objectifs du projet sont fondamentaux. Dans les cas d'EC n'impliquant qu'une organisation, cette phase est simplifiée par la sélection, au titre de coordinateur du projet, d'un responsable du programme, membre du personnel ou pair éducateur ou prestataire particulièrement motivé.

Dès le moment où les principaux partenaires s'entendent sur les objectifs, le coordinateur du projet doit informer les organisations communautaires de l'exercice envisagé et les inviter à participer. Le coordinateur explique généralement que son organisme parraine une activité de renforcement de capacités dont les résultats produiront une information intéressante et utile quant aux bénéficiaires du programme. L'exercice n'impose aucun coût à l'organisation. II suffit à cette dernière d'envoyer tous leurs pairs éducateurs ou autres prestataires (ou un sous-groupe représentatif) à un atelier de formation et d'être disposée, en plus de ses responsabilités habituelles, à collecter les données demandées pendant la période de l'étude (quatre à six semaines). Un à deux mois après la collecte des données, les organisations participantes seront invitées à une nouvelle réunion. Les résultats de l'étude leur seront présentés et l'occasion leur sera donnée d'exprimer leurs réactions et commentaires. 
Si les responsables d'un programme déclinent une invitation à participer - parce qu'ils estiment leur personnel trop occupé, craignent d'être jugés par l'EC ou pour toute autre raison -, il sera bon pour le coordinateur du projet de les inviter quand même à l'atelier de dissémination. Ces responsables auront ainsi l'occasion de juger pour eux-mêmes de l'utilité de l'exercice de couverture.

Dans les cas où une organisation communautaire effectuerait elle-même un exercice de couverture, il peut être désirable de former tous les pairs éducateurs à la collecte d'information, plutôt que de n'en sélectionner que quelques-uns. En l'occurrence, un responsable ou administrateur du programme assumerait vraisemblablement le rôle de coordinateur, en partenariat peut-être, avec un organisme d'assistance technique lors de la première mise en œuvre. L'EC produirait une information relative à la fois à l'état général du programme et aux accomplissements individuels des pairs éducateurs. Le résultat représenterait en somme une auto-évaluation de chaque pair éducateur et le début d'un système d'information de gestion (SIG). Dès le moment où l'organisation communautaire a accompli un premier EC, elle peut poursuivre la collecte et l'analyse régulières de données relatives à ses services.

\section{Avantages de la participation à un exercice de couverture}

- renforcement des capacités individuelles et institutionnelles

- le personnel du programme apprécie généralement les formations offertes et les considère utiles

- auto-évaluation des personnes en contact direct avec les bénéficiaires du programme

- fondement d'un système d'information de gestion au niveau du programme

- identification des bénéficiaires touchés, et par quels services

\section{Raisons pour lesquelles les organisations hésitent parfois à participer}

- crainte pour les responsables d'être jugés

- souci de surmenage du personnel

Le coordinateur doit prendre contact avec les organismes participants quelques jours avant l'atelier, afin d'y confirmer la présence des pairs éducateurs ou prestataires. 
L'équipe devra entre-temps identifier :

- Un coordinateur d'analyse sachant utiliser le logiciel statistique ; si une seule organisation participe à l'EC, ce coordinateur sera appelé à produire de simples tableaux croisés, à l'aide d'un logiciel statistique ou manuellement, pour l'analyse des données. Durée totale estimée de la tâche : un mois

- Un responsable adjoint de projet pour chaque groupe de cinq organisations participantes. Idéalement, les responsables adjoints devront maîtriser les langues parlées dans les zones de collecte des données. (Facultatif mais désirable.) Durée totale estimée de la tâche : 3 semaines

- Deux ou trois assistants chargés de la saisie et du nettoyage des données (peut aussi être le coordinateur chargé de l'analyse). Durée totale estimée de la tâche : 3 à 5 semaines, suivant la quantité de données attendues

- Un formateur chargé de la conduite de l'atelier - idéalement, cette personne sera la même que le responsable adjoint du projet. Durée totale estimée de la tâche : 3 jours (2 jours de préparation, 1 jour d'atelier)

Pour les EC pris en charge par un organisme d'assistance technique, les services assurent la répartition des tâches entre le coordinateur d'analyse, les responsables adjoints du projet et les assistants chargés de la saisie des données. Dans le cas des organisations communautaires qui entreprennent elles-mêmes un EC, le personnel existant peut assumer les fonctions prescrites ou il peut être nécessaire pour certains rôles de faire appel à des consultants extérieurs.

Les centres de jeunesse, qui partagent généralement un espace intérieur et extérieur communs, peuvent être amenés à faire appel à un personnel supplémentaire. Les centres de jeunes populaires attirent en effet parfois des centaines d'adolescents par jour. Elles ne disposent cependant généralement que d'un personnel limité : un responsable du centre et un personnel (rémunéré ou bénévole) juste suffisant à assurer une présence adulte parmi les jeunes. Cette combinaison de facteurs complique légèrement la tâche pour le personnel des centres de jeunes, par rapport aux pairs éducateurs. Il est possible d'optimiser l'enregistrement du nombre de jeunes fréquentant un centre de jeunes en plaçant un membre du personnel à la sortie de l'établissement. Cette personne peut inscrire les jeunes dans le registre chaque fois qu'ils quittent le centre pendant la période de l'étude. L'approche n'est cependant pas toujours pratique. 
Une autre approche consiste dès lors à engager, pour les besoins du projet, un ou deux assistants par centre, chargés d'enregistrer les jeunes à la sortie de l'établissement. Suivant la méthode retenue, la phase de planification peut exiger l'embauche et la formation d'assistants au projet. Les personnes chargées de la collecte des données sont celles qu'il convient d'envoyer à l'atelier de formation.

\section{Finalisation de l'outil de collecte des données (registre d'activité)}

Une fois l'équipe de recherche en place, la préparation à l'atelier de formation s'impose. Une étape essentielle en est la préparation des formulaires de collecte de données (les registres d'activité), en collaboration avec les pairs éducateurs et autres prestataires, leurs responsables ou les deux. Le formulaire le plus simple et le plus rapide de cet outil est présenté à l'annexe A.1. Sous ce format élémentaire, le formulaire se prête aux calculs manuels et ressemble davantage à un registre d'entrée/sortie. Pour entamer le processus de collecte des données, nous proposons un formulaire de collecte d'information sur les pairs éducateurs eux-mêmes (Annexe B.1) et un questionnaire que les organisations peuvent remplir dès le départ (Annexe B.2). L'annexe B.3 est un exemple de registre d'activité que les pairs éducateurs peuvent utiliser pour leurs activités en face à face (ce formulaire est inspiré de celui utilisé lors de l'EC en Éthiopie). Le registre illustré à l'annexe B.4 est également destiné aux pairs éducateurs, pour les activités de groupe cette fois (ce registre est également inspiré de l'EC d'Éthiopie). L'annexe B.5 propose un registre d'activité type à utiliser à l'entrée ou à la sortie d'un centre de jeunes (inspiré du formulaire utilisé en Mauritanie). Dans certains cas, il sera utile d'organiser une réunion antérieure à l'atelier (un semaine ou même un jour seulement avant la formation), pour la discussion du formulaire de collecte des données et les ajustements à faire en tenant compte des contextes national et local. II importe de considérer le moment (par rapport aux vacances scolaires, jours fériés, événements spéciaux, etc.) et la durée de la période de collecte des données. La durée variera (de 1 à 12 semaines) suivant le contexte, les ressources financières et l'évaluation des changements dans le temps. Si vous essayez d'obtenir un « instantané » des services proposés, la collecte pourra se limiter à une ou deux semaines. Pour dresser un tableau plus complet de la situation, il pourra être utile de prolonger la collecte en vue d'une information plus généralement exacte. 


\section{(1) Population Council}

La structure de base du formulaire restera constante d'un contexte à l'autre, mais il est important d'en assurer la pertinence locale des termes. Ainsi, la catégorie relative à l'état matrimonial devra peut-être être modifiée suivant le contexte : les termes « concubinage », « cohabitation » ou « relation à long terme » reflètent en effet parfois mieux le contexte social que les adjectifs « célibataire » ou « marié/e ». Il est essentiel que les formulaires reflètent la portée exacte du travail des pairs éducateurs et autres prestataires. Outre les simples registres d'activité, les formulaires de collecte d'information sur les pairs éducateurs (Annexe B.1) et les organisations elles-mêmes (Annexe B.2) apportent une information contextuelle précieuse. On veillera à ce que les pairs éducateurs indiquent leur nom et organisation, de sorte que les collecteurs de données puissent les associer correctement à leurs organisations et registres d'activité correspondants. N'oubliez pas qu'il importe de noter si les garçons parlent aux garçons, les filles aux filles, etc.

\section{Préparation à l'atelier de formation}

L'ordre du jour de la formation du Burkina Faso est joint à l'annexe C. Outre l'ordre du jour, la préparation des fournitures nécessaires à la recherche et la reproduction des registres d'activité sont importantes. L'atelier terminé, les pairs éducateurs et autres prestataires s'en retourneront dans leurs communautés respectives prêts à entreprendre la collecte des données. Ils devront par conséquent quitter l'atelier tout matériel nécessaire en mains, à savoir :

- Formulaires de collecte en suffisance pour toute la période d'étude. Au Burkina Faso, chaque pair éducateur a reçu suffisamment de formulaires pour enregistrer jusqu'à 300 contacts. Cette réserve s'y est avérée suffisante. Elle ne le sera pas nécessairement dans d'autres contextes. Les pairs éducateurs n'auront pas nécessairement idée du nombre de bénéficiaires avec lesquels ils entrent en contact en l'espace d'un mois (il s'agit, précisément, de l'une des raisons d'exécution d'un EC). Il convient dès lors de trouver l'équilibre entre l'apport aux pairs éducateurs d'un nécessaire de collecte suffisant mais pas trop encombrant ou intimidant. Au Burkina Faso, chaque pair éducateur a par exemple reçu 60 registres d'activité individuels (Annexe B.3) et 20 registres d'activités de groupe (Annexe B.4). Lors de leurs visites sur le terrain, les responsables adjoints du projet ont veillé à se munir de formulaires supplémentaires pour renflouer les réserves éventuellement épuisées.

- Stylos à bille nécessaires pour remplir les formulaires. 


\section{(2) Population Council}

- Matériel complémentaire (liste des codes à utiliser s'ils ne figurent pas sur les formulaires, coordonnées des membres de l'équipe, etc.)

- Classeurs utiles à l'organisation des formulaires et à la facilité du transport.

\section{Calendrier}

L'équipe doit fixer le calendrier de l'exercice de couverture, de l'atelier de formation à la phase 3 incluse (voir ci-dessous).

Elle doit également décider de la manière dont elle récupérera les formulaires de collecte en fin d'exercice. Les pairs éducateurs et autres prestataires viennent parfois de régions rurales distantes pour assister à la formation donnée dans la capitale du pays. II serait probablement irréaliste toutefois, pour raisons de temps comme de ressources monétaires, de les faire revenir en fin de période de collecte pour remettre leurs formulaires au responsable du projet. Dans la mesure du possible, il est certainement idéal de recevoir les registres complétés de la main propre des pairs éducateurs, ou de celle d'un pair éducateur désigné pour recueillir tous les registres de son groupe régional et les retourner ensuite au centre d'analyse. Si les circonstances ne le permettent pas, d'autres options peuvent être discutées avant le lancement de l'EC. Dans certains contextes, il peut être pratique ou efficace de dépêcher un responsable adjoint du projet de site en site pour la collecte des formulaires. Certains endroits disposent de systèmes de messagerie officieux : cette solution s'est avérée utile au Burkina Faso. En fin de compte, les partenaires locaux sont les mieux placés pour déterminer les meilleures modalités de transport des données, de manière efficace et sûre depuis les régions rurales jusqu'aux centres de traitement. 


\section{Phase 3. Formation et lancement de la collecte de données}

- Durée estimée : 4 à 6 semaines (ou plus longtemps si la période de collecte va jusqu'à 12 semaines)

\section{- Principales activités accomplies:}

- formation des pairs éducateurs ou autres prestataires à la collecte d'information sur leurs activités propres et celles des personnes avec lesquelles ils entrent en contact

- mise en place des responsables adjoints au soutien de la collecte des données

- collecte des formulaires remplis

- configuration des écrans de saisie des données

\section{Atelier de formation}

L'atelier de formation exige une journée (dans les endroits à accès limité, il peut être nécessaire de prévoir une journée supplémentaire, pour les besoins de déplacement des participants). Généralement, le coordinateur présente la justification de l'EC et le formateur explique ensuite aux pairs éducateurs et autres prestataires participants la manière d'aborder le formulaire de collecte des données. Le formateur décrit, colonne par colonne, l'information à recueillir et les différentes manières de poser les questions sur le terrain. II passe en revue les directives de confidentialité (il n'est pas nécessaire d'enregistrer le nom de qui que ce soit) et le but de l'évaluation du programme, y compris la manière dont les données seront utilisées. Une ample période de questionsréponses et de dialogue entre le formateur et les participants est prévue. Après l'explication initiale, les pairs éducateurs ou autres prestataires se familiarisent avec le formulaire de collecte d'information par jeux de rôles. Le formateur et les responsables adjoints circulent dans la salle pour s'assurer, en face à face ou par petits groupes, que chacun comprend bien le mode d'utilisation du formulaire et peut répondre aux questions. Une fois ces compétences maîtrisées (il convient de prévoir jusqu'à une heure pour cette partie de la formation), un autre formulaire est présenté. Destiné à saisir la même information, ce second formulaire est conçu pour faciliter la collecte dans les groupes plus vastes. Ici encore, le formateur décrit le formulaire et les pairs éducateurs ou autres prestataires s'exercent à le remplir les uns avec les autres. Le formateur mettra l'accent sur les points suivants : 


\section{(1) Population Council}

- Le formulaire doit être rempli pour chacune des personnes avec lesquelles le pair éducateur ou prestataire entre en contact, même si la personne est plus âgée ou plus jeune que la tranche d'âge visée et même si la rencontre a lieu dans dans une situation de groupe.

- II n'est pas nécessaire d'enregistrer le nom de la personne - toutes les données recueillies sont confidentielles.

- Si une personne préfère ne pas être inscrite dans le registre d'activité, elle ne doit pas l'être (inscrire NA en début de ligne et passer à la ligne pour la personne suivante).

- Dans la liste d'activités, il est essentiel d'indiquer toutes celles applicables à un échange particulier - les réponses multiples sont acceptables ; la même règle s'applique aux thèmes couverts. (II convient de consacrer un peu plus de temps à cette partie lors du jeu de rôles.)

- Les registres d'activité doivent toujours être remplis à l'encre.

- Le registre est conçu pour prendre note des clients habituels ; il convient d'enregistrer leurs échanges avec les mêmes personnes, même s'ils parlent chaque jour aux mêmes personnes. Ce type d'information est extrêmement précieux!

- Il convient de ne pas planifier d'activités spéciales durant la période de collecte des données, mais, plutôt, de mener les activités ordinaires.

- Les pairs éducateurs ou autres prestataires doivent être les seuls à ajouter des noms au registre. S'ils animent un groupe, ils peuvent demander à un assistant de parcourir les lieux pour remplir le registre d'activité du groupe.

- Il convient d'avoir toujours sur soi les registres d'activité, pendant toute la durée de la période de collecte.

- Les questions ne doivent être posées qu'après un échange avec le pair. Le questionnaire est bref et ne devrait pas prendre plus de deux minutes. La formation doit insister sur le fait que cette activité ne doit pas perturber le rôle habituel du pair éducateur ou autre prestataire. Le questionnaire ne doit pas affecter la qualité des rapports présents ou futurs avec le pair.

- Lors de la formation, il est utile de rappeler aux pairs éducateurs que l'exercice leur apporte, à eux, la possibilité de suivre tous leurs échanges au cours du mois considéré. C'est comme s'ils prenaient une photographie de ce qui se passe effectivement, de ce qu'ils accomplissent. C'est pour eux une occasion unique d'informer les autres sur la nature de leur travail.

- Tout au long de l'atelier, le formateur doit consacrer un temps adéquat aux questions et commentaires relatifs aux formulaires, pour s'assurer que chacun comprenne bien l'exercice et pour encourager aussi la participation et les 


\section{(2) Population Council}

échanges de commentaires. Certains pairs éducateurs peuvent par exemple se demander comment le pair comprendra une question. Le formateur expliquera toutes les questions et les réponses possibles à chacune. Avant la fin de l'atelier, le formateur s'assurera qu'il n'y a plus de questions, que les participants ont bien compris comment remplir le formulaire et qu'ils sont prêts à entreprendre la collecte sur le terrain.

- Le formateur pourra suggérer une ou deux phrases d'introduction dans le guide d'instructions de l'EC, pour aider les pairs éducateurs ou autres prestataires à expliquer brièvement leur intervention aux pairs interrogés. Ils pourront ainsi lire cette phrase avant de remplir le registre d'activité en présence du pair. Par exemple : «Est-ce que je pourrais te poser quelques questions ? Je fais un rapport sur tous les contacts que j'ai avec mes pairs, pour que l'association puisse se faire une meilleure idée de ce que représente mon travail. Les pairs éducateurs font de même dans tout le pays, ce mois-ci. Tu n'es pas obligé/e de me donner ton nom. »

Les pairs éducateurs ou autres prestataires doivent quitter l'atelier dotés de tout le matériel et de toute l'information dont ils ont besoin, y compris les coordonnées des responsables adjoints du projet (le cas échéant). Il leur est demandé d'entamer la collecte de données dès leur retour chez eux et la reprise de leurs activités et de ne pas hésiter à appeler le coordinateur du projet pour toute question qu'ils pourraient se poser. Dans certains contextes, si le budget de l'exercice le permet, il peut être désirable de fournir une carte téléphonique aux pairs éducateurs.

\section{Assistance des responsables adjoints aux pairs éducateurs ou autres prestataires}

Quelques mots sur les responsables adjoints et la décision d'en désigner ou non : Le rôle des responsables adjoints consiste, durant la phase de collecte des données, à assister les pairs éducateurs ou autres prestataires à la tâche, en se tenant à leur disposition, par téléphone ou en personne. Les pairs éducateurs sont les mieux placés pour faire face aux différentes situations qu'ils rencontrent sur le terrain et peuvent, au besoin, se réapprovisionner facilement en formulaires. Les responsables adjoints ne sont pas indispensables mais ils contribuent à la collecte de données de qualité. Si les responsables des programmes ont assisté à l'atelier de formation, ils peuvent assumer eux-mêmes ce rôle d'assistance aux pairs éducateurs. Suivant le contexte, un système d'assistance centralisé n'est pas nécessairement requis. Ainsi, si une organisation tout entière fait participer ses pairs éducateurs à un EC, ils peuvent s'entraider eux-mêmes. 
Il y aurait dans ce cas un contact direct intégré avec un responsable et une équipe d'étude.

S’ils sont engagés, les responsables adjoints peuvent s'acquitter de leurs responsabilités en téléphonant régulièrement aux pairs éducateurs et/ou en rendant visite à chacun au moins une fois pendant la période de collecte des données (ils se déplaceront en autobus, en train ou en taxi de brousse et logeront dans de petits hôtels). Plusieurs sites seront combinés par voyage, de sorte que le responsable adjoint pourra passer deux semaines d'affilée sur le terrain, se rendant de site en site.

\section{Collecte des formulaires de données}

Au terme de la période de collecte de données désignée, les registres d'activité seront renvoyés - selon la méthode convenue - au coordinateur du projet.

\section{Configuration des écrans de saisie des données}

Durant la période de collecte des données, le coordinateur d'analyse préparera un écran de saisie destiné à faciliter la saisie ultérieure des données. Un programme logiciel tel qu'Epi Info peut être utilisé. Le coordinateur d'analyse supervisera également la récupération des formulaires de données des différents sites et assurera leur gestion lors de leur arrivée. Il ou elle enregistrera chaque formulaire reçu et créera un code liant anonymement un ensemble de formulaires à un pair éducateur et/ou à une organisation particulière. À ce stade, le coordinateur formera le personnel de saisie à la saisie des données sur l'écran configuré.

\section{Phase 4. Saisie et analyse des données}

\section{- Durée estimée : 4 à 6 semaines}

- Principales activités accomplies:

- saisie des données dans le logiciel et analyse, ou tabulation manuelle et analyse

- production des résultats préliminaires et présentation à la discussion 


\section{(2) Population Council}

\section{Saisie et analyse des données}

Le coordinateur d'analyse supervisera la saisie et le nettoyage des données, au moyen du logiciel Epi Info ou d'un autre progiciel statistique, ou par tabulation manuelle. Une organisation communautaire peut aussi charger un membre de son personnel, ou une petite équipe, de la compilation des résultats de l'EC. La sélection d'une approche ou de l'autre dépend de la capacité de l'organisation responsable de la mise en œuvre de l'EC. L'annexe $D$ présente une série de tableaux et figures produites dans le cadre d'exercices de couverture antérieurs. II convient de conserver les formulaires de données en lieu sûr jusqu'à conclusion des analyses et vérification complète des données (jusqu'à un an).

L'analyse doit :

- $\quad$ toujours stratifier par sexe, et généralement aussi par tranche d'âge, selon les regroupements définis dans les EDS (10 à 14 ans, 15 à 19 ans) ou en fonction de plus petites tranches telles que 10 à 12 ans, 13 à 15 ans et 16 à 19 ans ;

- se référer aux tableaux d'EDS désagrégés, ou à des tableaux comparables, si le pays en dispose. (Voir le site Web du Population Council, http://www.popcouncil.org/gfd/gfddhs.html, pour une liste de pays et toute information complémentaire, si de plus récentes données ou analyses complémentaires sont désirées pour un pays particulier, ou pour demander une analyse de pays non encore inclus) ;

- $\quad$ procéder par organisation, type d'organisation (organisations financées par un donateur particulier) et milieu (urbain/rural) d'activité ;

- $\quad$ tenir compte de l'état matrimonial ;

- $\quad$ tenir compte de l'état de scolarisation ;

- tenir compte des caractéristiques du pair éducateur ou autre prestataire (répondre aux questions : Les pairs éducatrices tendent-elles à parler davantage aux filles et les pairs éducateurs masculins, davantage aux garçons ? Quel âge ont les pairs éducateurs par rapport aux jeunes auxquels ils s'adressent ?) ;

répondre aux questions suivantes:

- Combien de thèmes les pairs éducateurs abordent-ils dans le cadre d'un échange type ?

- Quel est le thème, ou la combinaison de thèmes, qui réapparaît le plus souvent dans les échanges avec les pairs éducateurs? Le ou les thèmes abordés varient-ils suivant le sexe du pair et du pair éducateur? 
- Quelles sont les caractéristiques socio-démographiques des pairs atteints par les pairs éducateurs (sexe, âge, niveau scolaire le plus élevé atteint, état matrimonial, habitat, activité rémunératrice) ?

- Où l'échange a-t-il lieu (centre/maison de jeunes, clinique, école, champs, marché, terrain de football/basketball, association communautaire ou autre endroit spécifié) ?

- Si l'échange a lieu lors d'une activité particulière, quelle est la nature de cette activité (petit/grand groupe de discussion, discussion individuelle, questions-réponses, sports, art dramatique, spectacle vidéo, consultations d'orientation, distribution de pilules, distribution de préservatifs, conseil, traitement médical) ?

Quelques exemples de tableaux et figures d'analyse sont illustrés à l'annexe D.

\section{Quelques résultats de l'exercice de couverture réalisé en Éthiopie}

L'exercice de couverture en Éthiopie a été mené sur une période de six semaines auprès de 13 des plus grandes organisations pour les jeunes du pays. Les résultats ont notamment révélé que :

- Les programmes touchent davantage les garçons et jeunes hommes que les filles, près de 60 \% des contacts établis l'étant avec des garçons ou des hommes.

- Les garçons plus âgés et les hommes dominent les programmes, à concurrence de $45 \%$ de contacts avec des garçons de 15 ans et plus, et de 1 sur 5 avec des hommes âgés de 20 ans et plus. Quatorze pour cent des contacts établis l'ont été avec des répondants au-delà de l'âge désiré de 24 ans.

- Les programmes touchent la population scolarisée plus avantagée alors que la plupart des adolescents éthiopiens ne fréquentent pas l'école.

- L'apport d'information sur le VIH/sida est le service le plus fréquent, atteignant $73 \%$ des garçons et $64 \%$ des filles. Une analyse approfondie du contenu du message peut être nécessaire, $15 \%$ seulement des filles et $7 \%$ seulement des garçons ayant reçu une information sur les questions de genre, et $23 \%$ seulement des garçons (généralement plus âgés) ayant reçu une information sur le préservatif.

- Seuls $3 \%$ des filles abordées étaient mariées alors que la majorité des Éthiopiennes se marient en cours d'adolescence et que la plupart des filles sexuellement actives sont mariées.

Source : Mekbib, Erulkar et Belete, 2005.

Résultats disponibles en ligne (en anglais) à

http://www.cih.uib.no/journals/EJHD/eihd19-no1/60.Who\%20are\%20the\%20targets\%20of\%20youth\%20programs.pdf 
On notera, dans l'exemple éthiopien ci-dessus, un biais significatif des ressources vers les jeunes des tranches d'âge supérieures scolarisés, de sexe souvent masculin, ainsi qu'une réorientation du profil des services offerts dans les maisons de jeunes vers le VIH/sida, alors que de nombreux autres types d'information (compétences de vie, questions de genre et relations) semblent laissées pour compte. La tendance est aussi apparente dans les données du Burkina Faso, où les rapports indiquent que la majorité de l'information reçue par les contacts concerne le $\mathrm{VIH} /$ sida (21\% des garçons et $16 \%$ des filles), tandis que les autres thèmes reçoivent beaucoup moins d'attention ( $y$ compris l'information relative aux compétences de vie, reçue par $5 \%$ seulement des garçons et $3 \%$ des filles) (Population Council et UNFPA, 2005).

\section{Présentation des résultats préliminaires pour dissémination}

Une fois l'analyse complète, le coordinateur du projet préparera un rapport facile à comprendre et, si possible, un exposé visuel d'accompagnement à présenter d'abord aux pairs éducateurs ou autres prestataires et aux responsables des programmes, puis aux autres parties prenantes.

Cette présentation devra servir de point de départ à l'analyse, pour engager les responsables des programmes et ceux de leur mise en œuvre, dans le processus de réflexion quant à leurs bénéficiaires. La présentation doit chercher à comparer et contraster les données nationalement représentatives d'EDS avec les résultats de I'EC. Par exemple, les données d'EDS désagrégées dans un contexte particulier peuvent indiquer que $78 \%$ des filles de 15 à 19 ans ne sont pas scolarisées. L'EC indique quant à lui que $78 \%$ des filles de 15 à 19 ans qui ont recours au service offert par une organisation communautaire sont scolarisées. Cette comparaison aidera l'organisation communautaire à tirer la conclusion qu'elle ne touche qu'une proportion faible et spéciale des filles de 15 à 19 ans, étant donné que la majorité des filles ne sont pas scolarisées mais que la majorité des participantes à leur programme le sont. Les résultats doivent aussi être présentés dans le contexte de la ou des populations cibles du ou des programmes.

Durant cette phase, le coordinateur du projet doit procéder à l'organisation d'un atelier de dissémination à l'intention des responsables de programme et des pairs éducateurs. 


\section{Phase 5. Dissémination des données et réévaluation des programmes et politiques en fonction de l'éducation par les pairs}

Durée estimée: 1 à 2 jours par dissémination ; l'ajustement des programmes et politiques est permanent

- Principales activités accomplies:

- atelier de dissémination à l'intention des pairs éducateurs, prestataires et responsables de programme

- atelier de dissémination à l'intention des donateurs, organismes gouvernementaux et décideurs politiques

- révision des programmes pour mieux saisir les destinataires insuffisamment touchés par les modèles existants - ou détermination d'autres méthodes d'accès à ceux et celles qui ne participent pas aux programmes

\section{Atelier de dissémination à l'intention des pairs éducateurs, prestataires et responsables de programme}

Les pairs éducateurs - et autres prestataires ayant assuré la collecte des données - et leurs responsables sont les premiers à voir les analyses. Les données obtenues à la fois de l'EDS et de l'EC doivent être présentées sous forme claire et simple, aisément compréhensible au groupe, de manière à ce qu'ils puissent les interpréter par euxmême. Un animateur peut guider la discussion concernant la situation reflétée par les données et les analyses supplémentaires éventuellement justifiées et demandées par les organisations. Les raisons des résultats obtenus et les stratégies aptes à les améliorer et à accroître la couverture du programme doivent également être discutées. Le moment approprié pour la discussion de l'institutionnalisation de l'EC. Bien informé de l'état actuel de la couverture, le personnel du programme est à même de décider du meilleur moyen d'institutionnaliser l'usage de l'exercice pour la gestion et le suivi de ses opérations futures.

\section{Atelier de dissémination à l'intention des donateurs, organismes gouvernementaux, décideurs politiques et autres parties prenantes}

Tirant parti des réponses et recommandations des pairs éducateurs et responsables de programme, un nouveau rapport peut être préparé pour présentation à une seconde 
rencontre de dissémination. Cette deuxième rencontre, à l'intention des donateurs et des décideurs politiques, offre l'occasion de proposer de nouveaux modes d'accès aux groupes insuffisamment touchés et de rapport des insuffisances du programme existant. Aspect important à ce niveau, les groupes participants échangeront les leçons tirées du processus et les mesures prises pour améliorer leur couverture et l'accès aux destinataires insuffisamment touchés.

\section{Révision des programmes pour mieux saisir les destinataires insuffisamment touchés par les modèles existants - ou détermination d'autres méthodes d'accès à ceux et celles qui ne participent pas aux programmes}

Les résultats de l'exercice de couverture et les discussions aux ateliers de dissémination peuvent servir à renforcer ou à ajuster les modèles existants - ou en inspirer de nouveaux. Les changements potentiels envisageables sont à explorer immédiatement après que l'exercice de couverture ait eu lieu, tant que les idées sont encore fraîches et la motivation encore intense.

Ce guide d'information présente, à titre d'exemple, les outils et résultats d'exercices de couverture réalisés antérieurement. À ce jour, l'expérience laisse entendre que les prestataires, à tous les niveaux - au sein des organisations communautaires, organisations non gouvernementales, programmes gouvernementaux locaux et partenaires de mise en œuvre d'USAID --, peuvent mettre ce simple instrument au service du suivi et de l'évaluation de leurs programmes. L'issue de la collecte de données aide les prestataires et les responsables de programme à déterminer si leurs programmes atteignent ou non leurs bénéficiaires visés. Cette information est à son tour utile aux responsables de la mise en œuvre et aux donateurs, en ce qu'ils peuvent concentrer leurs activités et subventions sur l'accès aux destinataires insuffisamment touchés, tels que définis à la fois par leur niveau de vulnérabilité et leur pondération numérique. 


\section{Annexe A.1 : Formulaire de collecte de données pour tabulation manuelle}

\begin{tabular}{|c|c|c|c|c|c|c|c|c|}
\hline & $\begin{array}{l}\text { Nombre d'adolescents } \\
\text { de chaque catégorie vus } \\
\text { durant les } 30 \text { derniers } \\
\text { jours }\end{array}$ & $\begin{array}{c}\text { Nbre de } \\
10 \text { à } 14 \text { ans }\end{array}$ & $\begin{array}{c}\text { Nbre de } \\
15 \text { à } 19 \text { ans }\end{array}$ & Nbre de filles & $\begin{array}{l}\text { Nbre de } \\
\text { garçons }\end{array}$ & $\begin{array}{c}\text { Nbre } \\
\text { d'adolescents } \\
\text { scolarisés }\end{array}$ & $\begin{array}{c}\text { Nbre } \\
\text { d'adolescents } \\
\text { non scolarisés }\end{array}$ & $\begin{array}{c}\text { Nbre } \\
\text { d'adolescents } \\
\text { mariés }\end{array}$ \\
\hline \multicolumn{9}{|l|}{ Filles } \\
\hline $\begin{array}{l}\text { 1. Filles célibataires } \\
\text { de } 10 \text { à } 14 \text { ans, } \\
\text { scolarisées }\end{array}$ & $\mathbf{A}$ & $\mathbf{A}$ & & $\mathbf{A}$ & & $\mathbf{A}$ & & \\
\hline $\begin{array}{l}\text { 2. Filles célibataires } \\
\text { de } 10 \text { à } 14 \text { ans, } \\
\text { non scolarisées }\end{array}$ & B & B & & B & & & $\bar{B}$ & \\
\hline $\begin{array}{l}\text { 3. Filles célibataires } \\
\text { de } 15 \text { à } 19 \text { ans, } \\
\text { scolarisées }\end{array}$ & C & & $\mathbf{C}$ & $\mathbf{C}$ & & $\mathbf{C}$ & & \\
\hline $\begin{array}{l}\text { 4. Filles célibataires } \\
\text { de } 15 \text { à } 19 \text { ans, } \\
\text { non scolarisées }\end{array}$ & D & & D & D & & & D & \\
\hline $\begin{array}{l}\text { 5. Filles mariées* } \\
\text { de } 10 \text { à } 14 \text { ans }\end{array}$ & $\mathbf{E}$ & $\mathbf{E}$ & & $\mathbf{E}$ & & & $\mathbf{E}$ & $\mathbf{E}$ \\
\hline $\begin{array}{l}\text { 6. Filles mariées* } \\
\text { de } 15 \text { à } 19 \text { ans }\end{array}$ & $\mathbf{F}$ & & $\mathbf{F}$ & $\mathbf{F}$ & & & $\mathbf{F}$ & $\mathbf{F}$ \\
\hline \multicolumn{9}{|l|}{ Garçons } \\
\hline $\begin{array}{l}\text { 7. Garçons célibataires } \\
\text { de } 10 \text { à } 14 \text { ans, } \\
\text { scolarisés }\end{array}$ & G & G & & & G & G & & \\
\hline $\begin{array}{l}\text { 8. Garçons célibataires } \\
\text { de } 10 \text { à } 14 \text { ans, } \\
\text { non scolarisés }\end{array}$ & H & $\mathbf{H}$ & & & $\mathbf{H}$ & & $\mathbf{H}$ & \\
\hline $\begin{array}{l}\text { 9. Garçons célibataires } \\
\text { de } 15 \text { à } 19 \text { ans, } \\
\text { scolarisés }\end{array}$ & I & & I & & I & I & & \\
\hline $\begin{array}{l}\text { 10. Garçons célibataires } \\
\text { de } 15 \text { à } 19 \text { ans, } \\
\text { non scolarisés }\end{array}$ & $\mathbf{J}$ & & $\mathbf{J}$ & & $\mathbf{J}$ & & $\mathbf{J}$ & \\
\hline $\begin{array}{l}\text { 11. Garçons mariés } \\
\text { de } 15 \text { à } 19 \text { ans*** }\end{array}$ & $\mathbf{K}$ & & $\mathbf{K}$ & & $\mathbf{K}$ & & & $\mathbf{K}$ \\
\hline Total : & $\begin{array}{c}\mathrm{A}+\mathrm{B}+\mathrm{C}+\mathrm{D}+\mathrm{E}+\mathrm{F}+\mathrm{G}+\mathrm{H} \\
+\mathrm{I}+\mathrm{J}+\mathrm{K}\end{array}$ & $\mathrm{A}+\mathrm{B}+\mathrm{E}+\mathrm{G}+\mathrm{H}$ & $\begin{array}{c}\mathrm{C}+\mathrm{D}+\mathbf{F}+\mathbf{I}+\mathbf{J}+ \\
\mathbf{K}\end{array}$ & $\begin{array}{c}\mathrm{A}+\mathrm{B}+\mathrm{C}+\mathrm{D}+\mathrm{E} \\
+\mathrm{F}\end{array}$ & $\begin{array}{c}\mathbf{G + H + I + J +} \\
\mathbf{K}\end{array}$ & $\mathbf{A}+\mathbf{C}+\mathbf{G}+\mathbf{I}$ & $\begin{array}{c}\mathrm{B}+\mathrm{D}+\mathrm{E}+\mathrm{F}+\mathrm{H}+ \\
\mathrm{J}\end{array}$ & $\mathbf{E}+\mathbf{F}+\mathbf{K}$ \\
\hline
\end{tabular}

*Les adolescentes mariées sont rarement scolarisées ; leur état de scolarisation n’est dès lors pas indiqué.

**Les garçons de 10 à 14 ans sont rarement mariés ; ils ne sont par conséquent pas inclus dans cette feuille de calcul.

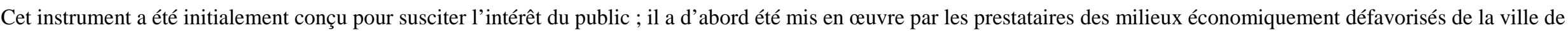

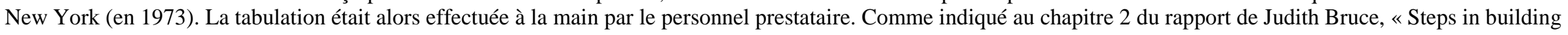

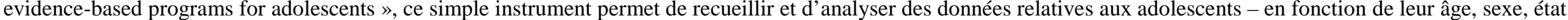
matrimonial et de scolarisation, habitat et autres variables pertinentes - par analyses de situation et autres méthodes de recherche (2003, p. 29). 


\section{(2) Population Council}

\section{Annexe A.2 - Feuille de calcul : Mappage de contenu des programmes pour adolescents}

\begin{tabular}{|c|c|c|c|c|c|c|c|}
\hline Catégories de contenu & $\begin{array}{l}\text { Groupe d'âge } \\
\text { concerné }\end{array}$ & Sexe* & Scolarisation $^{\Delta}$ & $\begin{array}{c}\text { Activités } \\
\text { touchant les } \\
\text { adolescents } \\
\text { mariés }\end{array}$ & $\begin{array}{c}\text { Nombres } \\
\text { touchés chaque } \\
\text { année }\end{array}$ & $\begin{array}{c}\% \text { de l'effort } \\
\text { programmatique } \\
\text { total }-20,40, \\
60,80,100 \%\end{array}$ & $\begin{array}{c}\text { Point d'entrée } \\
\text { clé ? }\end{array}$ \\
\hline \multicolumn{8}{|l|}{$\begin{array}{l}\text { Alphabétisme fonctionnel et } \\
\text { apprentissage hors-école }\end{array}$} \\
\hline \multicolumn{8}{|l|}{ Nutrition } \\
\hline \multicolumn{8}{|l|}{$\begin{array}{l}\text { Information en santé de la } \\
\text { reproduction }\end{array}$} \\
\hline \multicolumn{8}{|l|}{ Services de santé } \\
\hline \multicolumn{8}{|l|}{$\begin{array}{l}\text { Formation subsistance et } \\
\text { qualifications }\end{array}$} \\
\hline \multicolumn{8}{|l|}{$\begin{array}{l}\text { Possibilités d'épargne/ } \\
\text { alphabétisme économique }\end{array}$} \\
\hline \multicolumn{8}{|l|}{$\begin{array}{l}\text { Offre de protection/sécurité } \\
\text { aux adolescents vulnérables }\end{array}$} \\
\hline \multicolumn{8}{|l|}{$\begin{array}{l}\text { Encouragement de la } \\
\text { participation des adolescents } \\
\text { aux processus } \\
\text { communautaires et civiques }\end{array}$} \\
\hline \multicolumn{8}{|l|}{$\begin{array}{l}\text { Offre de possibilités de } \\
\text { renforcement de force } \\
\text { physique/sport et aptitudes } \\
\text { au jeu }\end{array}$} \\
\hline \multicolumn{8}{|l|}{$\begin{array}{l}\text { Établissement d'espaces } \\
\text { d'apprentissage et } \\
\text { d'épanouissement non } \\
\text { scolaires et non familiaux } \\
\text { pour les jeunes }\end{array}$} \\
\hline $\begin{array}{l}\text { * Exclusivement ou principal } \\
\text { Exclusivement ou principal } \\
\text { Plus ou moins mixte à parts }\end{array}$ & $\begin{array}{l}\text { nt filles - F } \\
\text { nt garçons - G } \\
\text { les -- M }\end{array}$ & & & $\begin{array}{l}\text { Participant } \\
\text { Participant } \\
\text { moins mix }\end{array}$ & $\begin{array}{l}\text { our la plupart sce } \\
\text { principalement } \mathrm{n} \\
\text { à parts égales -- }\end{array}$ & $\begin{array}{l}\text { olarisés -- S } \\
\text { on scolarisés -- N } \\
M\end{array}$ & \\
\hline
\end{tabular}

N.B.: Cette feuille de calcul et les zones programmatiques listées le sont à titre indicatif seulement. Dans un contexte particulier, ces catégories doivent refléter le contexte. 
Annexe B.1 : Fiche d'inscription des pairs éducateurs*

\begin{tabular}{|c|c|c|c|c|c|c|c|c|c|c|}
\hline $\begin{array}{l}(1) \\
N^{\circ}\end{array}$ & $\begin{array}{l}(2) \\
\text { Nom }\end{array}$ & $\begin{array}{c}\text { (3) } \\
\text { Organisation }\end{array}$ & $\begin{array}{c}\text { (4) } \\
\text { Quel } \\
\text { âge } \\
\text { as-tu? }\end{array}$ & $\begin{array}{c}(5) \\
\text { Es-tu } \\
\text { fille ou } \\
\text { garçon } \\
?\end{array}$ & $\begin{array}{c}6) \\
\text { Es-tu } \\
\text { inscrit/e } \\
\text { à } \\
\text { l'école? } \\
\text { (Oui/Non) } \\
\end{array}$ & $\begin{array}{c}\text { (7) } \\
\text { Quel est ton } \\
\text { niveau } \\
\text { d'études le } \\
\text { plus élevé? }\end{array}$ & $\begin{array}{c}\text { (8) } \\
\text { Vis-tu avec tes } 2 \\
\text { parents, mère } \\
\text { seule, père seul, } \\
\text { autres parents, } \\
\text { époux(se), aucun } \\
\text { parent, seul(e)? }\end{array}$ & $\begin{array}{c}\text { (9) } \\
\text { Es-tu } \\
\text { marié/e, } \\
\text { célibataire } \\
\text { jamais } \\
\text { mariéle, } \\
\text { séparéle, } \\
\text { divorcéle ou } \\
\text { veuf/veuve? }\end{array}$ & $\begin{array}{c}(10) \\
\text { Depuis } \\
\text { combien } \\
\text { de temps } \\
\text { es-tu pair } \\
\text { éducateur } \\
?\end{array}$ & $\begin{array}{c}\text { (11) } \\
\text { Quelle est ta } \\
\text { formation? } \\
\text { Utiliser les codes } \\
\text { ci-dessous. } \\
\text { Indiquer tous les } \\
\text { thèmes } \\
\text { applicables } \\
\text { (choix multiple). }\end{array}$ \\
\hline 1 & Annie & $\begin{array}{l}\text { Population } \\
\text { Council }\end{array}$ & 24 & Fille & Non & $4 e$ & 2 parents & $\begin{array}{c}\text { Célibataire } \\
\text { jamais } \\
\text { mariée }\end{array}$ & 3 mois & $1,3,8$ \\
\hline 2 & & & & & & & & & & \\
\hline 3 & & & & & & & & & & \\
\hline 4 & & & & & & & & & & \\
\hline 5 & & & & & & & & & & \\
\hline 6 & & & & & & & & & & \\
\hline 7 & & & & & & & & & & \\
\hline 8 & & & & & & & & & & \\
\hline 9 & & & & & & & & & & \\
\hline 10 & & & & & & & & & & \\
\hline 11 & & & & & & & & & & \\
\hline 12 & & & & & & & & & & \\
\hline 13 & & & & & & & & & & \\
\hline 14 & & & & & & & & & & \\
\hline 15 & & & & & & & & & & \\
\hline
\end{tabular}

* Ce formulaire doit être rempli par les pairs éducateurs.

CODES THÉMATIQUES :

\begin{tabular}{|l|l|l|}
\hline \hline 1=VIH/SIDA & 8=CONTRACEPTION & 12=DROGUE/ALCOOL \\
2=PLANIFICATION & D'URGENCE & 13=DÉLINQUANCE \\
FAMILIALE & 9=HYGIËNE & 14=QUESTIONS DE GENRE \\
3=IST & 10=COMPÉTENCES & 15=TRAITE D'ENFANTS \\
4=GROSSESSE & DE VIE & 16=MARIAGE \\
5=AVORTEMENT & 11=QUESTIONS/RELA & 17=DROITS DES ENFANTS \\
6=EXCISION & TIONS FAMILIALES & 18=AUTRE (PRÉCISER \\
7=PRÉSERVATIF & ET SOCIALES & DANS LE TABLEAU) \\
\hline
\end{tabular}


Annexe B.2 : Formulaire de collecte de données destiné aux organisations

1. Nom de l'organisation:

2. Adresse :

3. Dans quelles villes / régions œuvrez-vous ?

$$
\text { Urbain }
$$

Rural

Les 2

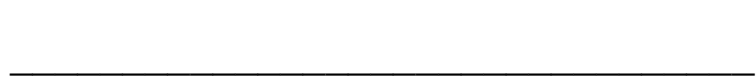

4. Combien de pairs éducateurs aviez-vous à votre service en 2005 ?

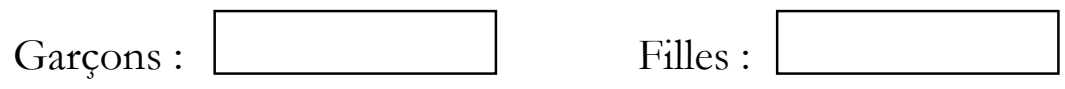

5. Quelle est la population cible de votre programme de pairs éducateurs ? Touchez-vous des sous-groupes particuliers (à l'école/hors-école, plus jeunes/plus âgés)?

6. Quels sont les thèmes de formation de vos pairs éducateurs ? (Veuillez indiquer toutes les réponses applicables.)

$\begin{array}{lll}\square \text { VIH/SIDA } & \square \text { IST } & \square \text { HYGIÈNE } \\ \begin{array}{l}\text { COMPÉTENCES } \\ \text { DE VIE }\end{array} & \square \text { PRÉSERVATIF } & \square \text { PLANIFICATION FAMILIALE } \\ \square \text { GENRE } & \square \text { GROSSESSE } & \square \text { CONTRACEPTION D'URGENCE } \\ \square \text { AVORTEMENT } & \square \text { DROGUE/ALCOOL } & \square \text { EXCISION } \\ \square \text { MARIAGE } & \square \text { DÉLINQUANCE } & \square \text { TRAITE D'ENFANTS } \\ \square \text { QUESTIONS/ } & \square \text { DROITS DES ENFANTS } & \square \text { AUTRE (PRÉCISER) } \\ \quad \text { RELATIONS } & \\ \text { FAMILIALES ET } & & \\ \text { SOCIALES } & \end{array}$

7. À quels types d'activités les pairs éducateurs participent-ils ?
DISCUSSION INDIVIDUELLE
DISCUSSION DE GROUPE
APPORT D'INFORMATION
ART DRAMATIQUE/THÉÂTRE
DISTRIBUTION DE
ORIENTATION VERS LES
PRÉSERVATIFS SERVICES CLINIQUES

AUTRE (préciser) 


\section{Annexe B.3 : Registre d'activité individuel pour pair éducateur ou prestataire}

(1) Nom du prestataire / pair éducateur

( 2 ) Organisation

Enregistrez chaque personne contactée sur une ligne.

\begin{tabular}{|c|c|c|c|c|c|c|c|c|c|c|c|c|c|c|}
\hline \multirow[t]{2}{*}{$\begin{array}{c}(3) \\
\text { Date }\end{array}$} & $\begin{array}{l}\text { (4) } \\
\text { Sexe }\end{array}$ & \begin{tabular}{c|c} 
(5) \\
Premier \\
contact avec le \\
programme?
\end{tabular} & $\begin{array}{c}\text { (6) } \\
\text { Déjà } \\
\text { enregistré ? }\end{array}$ & $\begin{array}{c}\text { (7) } \\
\text { Contact } \\
\text { individuel } \\
\text { ou } \\
\text { groupe? }\end{array}$ & \multicolumn{2}{|c|}{$\begin{array}{c}\text { (8) } \\
\text { Lieu de contact } \\
\text { (Utiliser les } \\
\text { codes-codes } \\
\text { multiples } \\
\text { admis) }\end{array}$} & $\begin{array}{c}\text { (9) } \\
\text { Type de } \\
\text { services } \\
\text { (Utiliser les } \\
\text { codes - codes } \\
\text { multiples } \\
\text { admis) }\end{array}$ & $\begin{array}{c}(10) \\
\text { Thèmes abordés } \\
\text { (Utiliser les codes } \\
\text { - codes multiples } \\
\text { admis) }\end{array}$ & $\begin{array}{c}(11) \\
\text { Inscrit/e } \\
\text { à } \\
\text { l'école? }\end{array}$ & $\begin{array}{c}(11) \\
\text { Plus haut niveau } \\
\text { scolaire atteint }\end{array}$ & $\begin{array}{c}(12) \\
\text { Activité } \\
\text { rémunératrice } \\
\text { durant le dernier } \\
\text { mois? }\end{array}$ & $\begin{array}{c}13) \\
\text { Habitat }\end{array}$ & $\begin{array}{c}(14) \\
\text { Etat } \\
\text { matrimonial }\end{array}$ & $\begin{array}{l}\text { (15) } \\
\text { Age }\end{array}$ \\
\hline & $\begin{array}{l}1=M \\
2=F\end{array}$ & $\begin{array}{l}1=\mathrm{OUI} \\
2=\mathrm{NON}\end{array}$ & $\begin{array}{l}1=\mathrm{OUI} \\
2=\mathrm{NON}\end{array}$ & $\begin{array}{l}\text { 1=INDIVID } \\
\text { 2=GROUPE }\end{array}$ & & & & & $\begin{aligned} 1 & =\mathrm{OUI} \\
2 & =\mathrm{NON}\end{aligned}$ & $\begin{array}{l}\text { 1. Aucun } \\
\text { 2. Primaire } \\
\text { 3. Secondaire } \\
\text { 4. Ens. coranique }\end{array}$ & $\begin{array}{l}1=\mathrm{OUI} \\
2=\mathrm{NON}\end{array}$ & $\begin{array}{l}\text { 1=2 parents } \\
\text { 2=Mère seule } \\
\text { =-Père seul } \\
\text { 4=Autres } \\
\text { parents } \\
5=\text { Époux/se } \\
6=\text { Aucun parent } \\
7=\text { Seul(e) }\end{array}$ & $\begin{array}{l}\text { 1=JAMAIS } \\
\text { MARIÉ/E } \\
\text { 2=MARIÉ/E } \\
\text { 3=SÉPARÉ/E } \\
\text { 4=DIVORCÉ/E } \\
\text { 5=VEUF/VEUV } \\
\text { E }\end{array}$ & \\
\hline & $\begin{array}{l}1=M \\
2=F\end{array}$ & $\begin{array}{l}1=\mathrm{OUI} \\
2=\mathrm{NON}\end{array}$ & $\begin{array}{l}1=\mathrm{OUI} \\
2=\mathrm{NON}\end{array}$ & $\begin{array}{l}\text { 1=INDIVID } \\
\text { 2=GROUPE }\end{array}$ & & & & & $\begin{aligned} 1 & =\mathrm{OUI} \\
2 & =\mathrm{NON}\end{aligned}$ & $\begin{array}{l}\text { 1. Aucun } \\
\text { 2. Primaire } \\
\text { 3. Secondaire } \\
\text { 4. Ens. coranique }\end{array}$ & $\begin{array}{l}1=O U I \\
2=N O N\end{array}$ & $\begin{array}{l}1=2 \text { parents } \\
2=\text { Mère seule } \\
\text { 3=Père seul } \\
\text { 4=Autres } \\
\text { parents } \\
5=\text { Époux/se } \\
6=\text { Aucun parent } \\
7=\text { Seul(e) }\end{array}$ & $\begin{array}{l}\text { 1=JAMAIS } \\
\text { MARIÉ/E } \\
\text { 2=MARIÉ/E } \\
\text { 3=SÉPARÉ/E } \\
\text { 4=DIVORCÉ/E } \\
\text { 5=VEUF/VEUV } \\
\text { E }\end{array}$ & \\
\hline & $\begin{array}{l}1=M \\
2=F\end{array}$ & $\begin{array}{l}1=\mathrm{OUI} \\
2=\mathrm{NON}\end{array}$ & $\begin{array}{l}1=\mathrm{OUI} \\
2=\mathrm{NON}\end{array}$ & $\begin{array}{c}1=\text { INDIVID } \\
2=\text { GROUPE }\end{array}$ & & & & & $\begin{aligned} 1 & =\text { OUI } \\
2 & =\text { NON }\end{aligned}$ & $\begin{array}{l}\text { 1. Aucun I } \\
\text { 2. Primaire } \\
\text { 3. Secondaire } \\
\text { 4. Ens. coranique }\end{array}$ & $\begin{array}{l}1=O U I \\
2=N O N\end{array}$ & $\begin{array}{l}1=2 \text { parents } \\
2=\text { Mère seule } \\
3=\text { Père seul } \\
4=\text { Autres } \\
\text { parents } \\
5=\text { Époux/se } \\
6=\text { Aucun parent } \\
7=\text { Seul(e) }\end{array}$ & $\begin{array}{l}\text { 1=JAMAIS } \\
\text { MARIÉ/E } \\
\text { 2=MARIÉ/E } \\
\text { 3=SÉPARÉ/E } \\
\text { 4=DIVORCÉ/E } \\
\text { 5=VEUF/VEUV } \\
\text { E }\end{array}$ & \\
\hline & $\begin{array}{l}1=M \\
2=F\end{array}$ & $\begin{array}{l}1=\mathrm{OUI} \\
2=\mathrm{NON}\end{array}$ & $\begin{array}{l}1=\mathrm{OUI} \\
2=\mathrm{NON}\end{array}$ & $\begin{array}{c}1=\text { INDIVID } \\
2=\text { GROUPE }\end{array}$ & & & & & $\begin{aligned} 1 & =\mathrm{OUI} \\
2 & =\mathrm{NON}\end{aligned}$ & $\begin{array}{l}\text { 1. Aucun } \\
\text { 2. Primaire } \\
\text { 3. Secondaire } \\
\text { 4. Ens. coranique }\end{array}$ & $\begin{array}{l}1=O U I \\
2=N O N\end{array}$ & $\begin{array}{l}1=2 \text { parents } \\
2=\text { Mère seule } \\
3=\text { Père seul } \\
4=\text { Autres } \\
\text { parents } \\
5=\text { Epoux/se } \\
6=\text { Aucun parent } \\
7=\text { Seul(e) }\end{array}$ & $\begin{array}{l}\text { 1=JAMAIS } \\
\text { MARIÉ/E } \\
\text { 2=MARIÉ/E } \\
\text { 3=SÉPARÉ/E } \\
\text { 4=DIVORCÉ/E } \\
\text { 5=VEUF/NEUV } \\
\text { E }\end{array}$ & \\
\hline \multicolumn{15}{|c|}{ CODES DE LIEU : } \\
\hline \multicolumn{2}{|c|}{$\begin{array}{l}1=\text { CENTRE } \\
\text { 2=CLINIQUE } \\
\text { 3=ÉCOLE } \\
\text { 4=QUARTIER } \\
\text { 5=CHEZ MOI } \\
\text { 6=MAISON DE } \\
\text { JEUNES } \\
\text { 7=MARCHÉ }\end{array}$} & $\begin{array}{l}\text { 8=CÉRÉMONIE } \\
\text { CAFÉ } \\
9=S T A D E \\
\text { 10=ASSOC. } \\
\text { COMMUNAUTAIRE } \\
\text { 11=AUTRE } \\
\text { (PRÉCISER DANS LE } \\
\text { TABLEAU) }\end{array}$ & \multicolumn{3}{|c|}{$\begin{array}{l}\text { 1= CONFÉRENCE } \\
\text { 2=DISCUSSION EN PETIT } \\
\text { GROUPE } \\
\text { 3=DISCUSSION EN TÊTE- } \\
\text { À-TÊTE } \\
\text { 4= QUESTIONS/RÉPONSES } \\
\text { 5= ART DRAMATIQUE } \\
\text { 6= SPECTACLE VIDÉO }\end{array}$} & \multicolumn{2}{|c|}{$\begin{array}{l}\text { 7= SPORTS } \\
\text { 8= DISTRIBUTION } \\
\text { DE PRÉSERVATIFS } \\
\text { 9= DISTRIBUTION } \\
\text { DE PILULES } \\
\text { 10= CONSEIL }\end{array}$} & $\begin{array}{l}\text { 11=ORIENTATION } \\
12=\text { TRAITEMENT } \\
\text { MÉDICAL } \\
\text { 13=AUTRE } \\
\text { (PRÉCISER DANS LE } \\
\text { TABLEAU) }\end{array}$ & \multicolumn{3}{|c|}{$\begin{array}{l}\text { 1=VIH/SIDA } \\
\text { 2=PLANIFICATION FAMILIALE } \\
\text { 3=IST } \\
\text { 4=GROSSESSE } \\
\text { 5=AVORTEMENT } \\
\text { 6=EXCISION } \\
\text { 7=PRÉSERVATIF } \\
\text { 8=CONTRACEPTION D'URGENCE } \\
\text { 9=HYGIËNE }\end{array}$} & \multicolumn{3}{|c|}{$\begin{array}{l}\text { 10=COMPÉTENCES DE VIE } \\
11=\text { QUESTIONS/RELATIONS FAMILIALES ET } \\
\text { SOCIALES } \\
12=\text { DROGUE/ALCOOL } \\
\text { 13=DÉLINQUANCE } \\
\text { 14=QUESTIONS DE GENRE } \\
\text { 15=TAITE D'ENFANTS } \\
\text { 16=MARIAGE } \\
\text { 17=DROITS DES ENFANTS } \\
\text { 18=AUTRE (PRÉCISER DANS LE TABLEAU) }\end{array}$} \\
\hline
\end{tabular}




\section{(1) Population Council}

Annexe B.4 : Fiche d'inscription pour activités de groupe des pairs éducateurs*

(1) Nom

( 2 ) Organisation.

(3) ID du pair éducateur

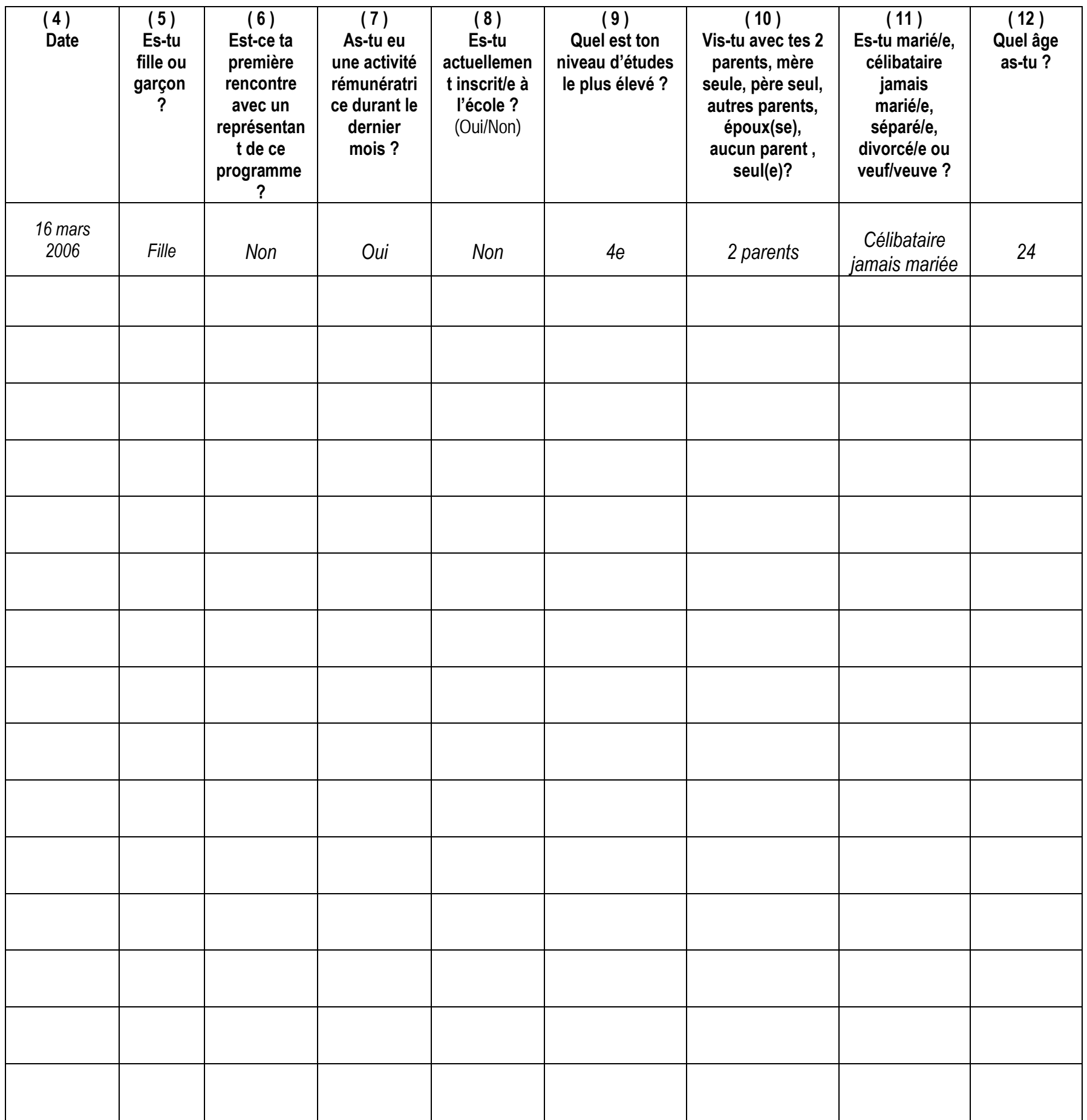

* Ce formulaire doit être utilisé lorsqu'un pair éducateur convoque un groupe. 
Annexe B.5 - Registre d'activité de centre de jeunes

(1) Nom du centre de jeunesse

Enregistrez chaque personne contactée sur une ligne.

\begin{tabular}{|c|c|c|c|c|c|c|c|c|c|c|c|c|}
\hline $\begin{array}{l}\text { (2) } \\
\text { Date }\end{array}$ & $\begin{array}{l}(3) \\
\text { Sexe }\end{array}$ & $\begin{array}{c}\text { ( } 4) \\
\text { Activités au centre } \\
\text { Utiliser les codes } \\
\text { ci-dessous. } \\
\text { Indiquer toutes le } \\
\text { activités citées } \\
\text { (choix multiple). }\end{array}$ & $\begin{array}{c}\text { ( } 5 \text { ) } \\
\text { As-tu participé } \\
\text { à une } \\
\text { discussion } \\
\text { avec un ami, } \\
\text { un pair } \\
\text { éducateur ou } \\
\text { le directeur/la } \\
\text { directrice? }\end{array}$ & $\begin{array}{c}(6) \\
\text { Thèmes discutés } \\
\text { aujourd'hui } \\
\text { Utiliser les } \\
\text { codes ci- } \\
\text { dessous. } \\
\text { Indiquer tous les } \\
\text { thèmes cités } \\
\text { (choix multiple). }\end{array}$ & $\begin{array}{c}\text { ( } 7 \text { ) } \\
\text { As-tu déjà } \\
\text { répondu à ce } \\
\text { questionnaire? }\end{array}$ & $\begin{array}{l}\text { ( } 8 \text { ) } \\
\text { Fréquence des } \\
\text { visites au } \\
\text { centre }\end{array}$ & $\begin{array}{c}\text { ( } 9 \text { ) } \\
\text { Es-tu } \\
\text { actuelle } \\
\text { ment } \\
\text { inscrit/e } \\
\text { à } \\
\text { l'école? }\end{array}$ & $\begin{array}{l}(10) \\
\text { Plus haut } \\
\text { niveau } \\
\text { scolaire } \\
\text { atteint }\end{array}$ & $\begin{array}{c}(11) \\
\text { Habitat }\end{array}$ & $\begin{array}{l}(12) \\
\text { Activité } \\
\text { rémuné } \\
\text { ratrice } \\
\text { durant } \\
\text { le } \\
\text { dernier } \\
\text { mois? }\end{array}$ & $\begin{array}{c}(13) \\
\text { État } \\
\text { matrimonial }\end{array}$ & $\begin{array}{c}(14) \\
\text { Âge }\end{array}$ \\
\hline & $\begin{array}{l}\text { 1. } \\
\text { Garçon } \\
\text { 2. Fille }\end{array}$ & & $\begin{array}{l}\text { 1. Non } \\
\text { (passer à [6]) } \\
\text { 2. Oui } \\
\text { (continuer) }\end{array}$ & & $\begin{array}{l}\text { 1. Non (passer à la } \\
\text { colonne suivante) } \\
\text { 2. Oui (FIN : Si oui, } \\
\text { passer le } \\
\text { questionnaire à la } \\
\text { personne suivante) }\end{array}$ & $\begin{array}{l}\text { 1. Une fois } \\
\text { 2. 2-3 fois } \\
\text { 3. 3-4 fois } \\
\text { 4. tous les jours } \\
\text { 5. moins d'une } \\
\text { fois par semaine } \\
\text { mais plus d'une } \\
\text { fois par mois }\end{array}$ & $\begin{array}{l}\text { 1. Oui } \\
\text { 2. Non }\end{array}$ & $\begin{array}{l}\text { 1. Nul } \\
\text { 2. Primaire } \\
\text { 3. } \\
\text { Secondaire } \\
\text { 4. Ens. } \\
\text { coranique }\end{array}$ & $\begin{array}{l}\text { 1=2 parents } \\
\text { 2=Mère seule } \\
\text { 3=Père seul } \\
\text { 4=Autres parents } \\
\text { 5=Époux/se } \\
6=\text { Aucun parent } \\
\text { 7=Seul(e) }\end{array}$ & $\begin{array}{l}\text { 1. Oui } \\
\text { 2. Non }\end{array}$ & $\begin{array}{l}\text { 1=JAMAIS } \\
\text { MARIÉ/E } \\
\text { 2=MARIÉ/E } \\
\text { 3=SÉPARÉ/E } \\
\text { 4=DIVORCÉ/E } \\
\text { 5=VEUF/VEUVE }\end{array}$ & \\
\hline & $\begin{array}{l}\text { 1. } \\
\text { Garçon } \\
\text { 2. Fille }\end{array}$ & & $\begin{array}{l}\text { 1. Non } \\
\text { (passer à [6]) } \\
\text { 2. Oui } \\
\text { (continuer) }\end{array}$ & & $\begin{array}{l}\text { 1. Non (passer à la } \\
\text { colonne suivante) } \\
\text { 2. Oui (FIN : Si oui, } \\
\text { passer le } \\
\text { questionnaire à la } \\
\text { personne suivante) }\end{array}$ & $\begin{array}{l}\text { 1. Une fois } \\
\text { 2. 2-3 fois } \\
\text { 3. 3-4 fois } \\
\text { 4. tous les jours } \\
\text { 5. moins d'une } \\
\text { fois par semaine } \\
\text { mais plus d'une } \\
\text { fois par mois }\end{array}$ & $\begin{array}{l}\text { 1. Oui } \\
\text { 2. Non }\end{array}$ & $\begin{array}{l}\text { 1. Nul } \\
\text { 2. Primaire } \\
\text { 3. } \\
\text { Secondaire } \\
\text { 4. Ens. } \\
\text { coranique }\end{array}$ & $\begin{array}{l}\text { 1=2 parents } \\
\text { 2=Mère seule } \\
\text { 3=Père seul } \\
\text { 4=Autres parents } \\
\text { 5=Époux/se } \\
6=\text { Aucun parent } \\
7=\text { Seul(e) }\end{array}$ & $\begin{array}{l}\text { 1. Oui } \\
\text { 2. Non }\end{array}$ & $\begin{array}{l}\text { 1=JAMAIS } \\
\text { MARIÉ/E } \\
\text { 2=MARIÉ/E } \\
\text { 3=SÉPARÉ/E } \\
\text { 4=DIVORCÉ/E } \\
\text { 5=VEUF/VEUVE }\end{array}$ & \\
\hline & $\begin{array}{l}\text { 1. } \\
\text { Garçon } \\
\text { 2. Fille }\end{array}$ & & $\begin{array}{l}\text { 1. Non } \\
\text { (passer à [6]) } \\
\text { 2. Oui } \\
\text { (continuer) }\end{array}$ & & $\begin{array}{l}\text { 1. Non (passer à la } \\
\text { colonne suivante) } \\
\text { 2. Oui (FIN : Si oui, } \\
\text { passer le } \\
\text { questionnaire à la } \\
\text { personne suivante) }\end{array}$ & $\begin{array}{l}\text { 1. Une fois } \\
\text { 2. 2-3 fois } \\
\text { 3. 3-4 fois } \\
\text { 4. tous les jours } \\
\text { 5. moins d'une } \\
\text { fois par semaine } \\
\text { mais plus d'une } \\
\text { fois par mois }\end{array}$ & $\begin{array}{l}\text { 1. Oui } \\
\text { 2. Non }\end{array}$ & $\begin{array}{l}\text { 1. Nul } \\
\text { 2. Primaire } \\
\text { 3. } \\
\text { Secondaire } \\
\text { 4. Ens. } \\
\text { coranique }\end{array}$ & $\begin{array}{l}\text { 1=2 parents } \\
\text { 2=Mère seule } \\
\text { 3=Père seul } \\
\text { 4=Autres parents } \\
\text { 5=Époux/se } \\
\text { 6=Aucun parent } \\
\text { 7=Seul(e) }\end{array}$ & $\begin{array}{l}\text { 1. Oui } \\
\text { 2. Non }\end{array}$ & $\begin{array}{l}\text { 1=JAMAIS } \\
\text { MARIÉ/E } \\
\text { 2=MARIÉ/E } \\
\text { 3=SÉPARÉ/E } \\
\text { 4=DIVORCÉ/E } \\
\text { 5=VEUF/VEUVE }\end{array}$ & \\
\hline
\end{tabular}

\section{CODES D'ACTIVITÉS AU CENTRE :}

\section{1=KARATÉ}

2=FOOTBALL

3=BASKETBALL

4=INFORMATIQUE/ORDINA

TEURS

5=RENCONTRE DE

SCOUTS

\section{CODES THÉMATIQUES :}

\section{$1=\mathrm{VIH} / \mathrm{SIDA}$}

2=PLANIFICATION

FAMILIALE

3=IST

4=GROSSESSE

$5=$ AVORTEMENT

6=EXCISION

7=PRÉSERVATIF

8=CONTRACEPTION
D'URGENCE
9=HYGIÈNE
10=COMPÉTENCES DE VIE
11=QUESTIONS/RELATIONS
FAMILIALES ET SOCIALES
12=DROGUE/ALCOOL

13=DÉLINQUANCE

14=QUESTIONS DE GENRE

15=TRAITE D'ENFANTS

16=MARIAGE

17=DROITS DES ENFANTS

18=AUTRE (PRÉCISER DANS LE

TABLEAU) 


\section{Annexe C : Ordre du jour de la formation au Burkina Faso \\ Qui les organisations de jeunes touchent-elles ? \\ Planification d'une activité d'exercice de couverture}

8 h -8 h 30

8 h $30-9$ h

9 h -9 h 15

9 h $15-9$ h 30

9 h $30-10$ h 15

10 h $15-10$ h 30

10 h $30-11$ h

11 h -11 h 15

11 h $15-12$ h 30

12 h $30-13$ h 15

$13 \mathrm{~h} 15-14 \mathrm{~h}$

$14 \mathrm{~h}-15 \mathrm{~h}$

$15 \mathrm{~h}-16 \mathrm{~h} 30$

16 h $30-17$ h
Inscriptions

Mots de bienvenue et introduction aux présentations

Objectifs de l'atelier

L'adolescence au Burkina Faso : quelques faits

Présentation du registre d'activité

Brève démonstration d'emploi par 2 participants - jeu de rôles

Pause

Jeux de rôles - utilisation des registres d'activité (tous participants)

Présentation du registre de groupe

Jeux de rôles - utilisation du registre de groupe (tous participants)

Déjeuner

Discussion et questions relatives aux jeux de rôles

(tous participants ; 1-2 animateurs)

Questions et distribution des classeurs

(tous participants ; 1-2 animateurs)

Autres questions et discussion

Conclusions et questions administratives 


\section{Annexe D : Exemples de résultats de l'exercice de couverture réalisé en Mauritanie}

(Source des tableaux et figures : UNPFA et Population Council,

Exercice de couverture de Mauritanie, 2006)

Tableau 1. Répartition selon le sexe des jeunes rencontrés dans les cinq centres de jeunes les plus actives durant la période de collecte des données (les données ont été collectées pendant une semaine dans chaque maison de jeunes)

\begin{tabular}{|c|c|c|c|}
\hline \multirow[t]{2}{*}{ Maison de jeunes } & \multicolumn{2}{|c|}{ Sexe } & \multirow[b]{2}{*}{ Nombre } \\
\hline & Masculin (\%) & Féminin $(\%)$ & \\
\hline A & 90 & 10 & 541 \\
\hline B & 90 & 10 & 403 \\
\hline $\mathrm{C}$ & 97 & 3 & 557 \\
\hline $\mathrm{D}$ & 51 & 49 & 385 \\
\hline $\mathrm{E}$ & 79 & 21 & 419 \\
\hline Total & 83 & 17 & 2305 \\
\hline
\end{tabular}

Comme l'indique le tableau 1, dans les centres de jeunes sauf un, beaucoup plus de garçons que de filles s'étaient rendus au centre.

Tableau 2 : Répartition (\%) des personnes s'étant rendues dans les centres de jeunes pendant la période de collecte des données, par âge et par sexe (tous centres de jeunes confondus)

\begin{tabular}{|l|c|c|c|c|c|c|c|l|}
\hline Centres de jeunes & \multicolumn{6}{|l|}{ Tranche d'âge (\%) } & \multirow{2}{*}{ Nombre } \\
\cline { 2 - 9 } & $<10$ & $10-14$ & $\mathbf{1 5}-\mathbf{1 9}$ & $20-24$ & $25-29$ & $30-34$ & $35+$ & \\
\hline Garçons & 4 & 28 & $\mathbf{4 2}$ & 15 & 5 & 2 & 3 & 4508 \\
\hline Filles & 4 & 28 & $\mathbf{4 5}$ & 15 & 5 & 2 & 1 & 944 \\
\hline Total & 4 & 28 & $\mathbf{4 2}$ & 15 & 5 & 2 & 3 & 5452 \\
\hline
\end{tabular}

Comme l'indique le tableau 2, les adolescents de 15 à 19 ans représentent près de la moitié des bénéficiaires accueillis dans les centres de jeunes. 
Tableau 3 : Répartition (\%) des personnes s'étant rendues dans les centres de jeunes en fonction du sexe et de la fréquence de visite hebdomadaire (tous centres de jeunes confondus)

\begin{tabular}{|l|c|c|c|c|}
\hline \multirow{2}{*}{$\begin{array}{c}\text { Nombre de visites } \\
\text { par semaine }\end{array}$} & \multicolumn{2}{|c|}{ Sexe } & Total & $\begin{array}{c}\text { Nomb } \\
\text { re }\end{array}$ \\
\cline { 2 - 3 } & Masculin & Féminin & & \\
\hline Une fois & $\mathbf{1 0}$ & $\mathbf{2 4}$ & $\mathbf{1 3}$ & $\mathbf{7 0 0}$ \\
2 à 3 fois & 27 & 32 & 28 & 1534 \\
4 à 5 fois & 17 & 15 & 17 & 922 \\
Tous les jours & $\mathbf{4 0}$ & $\mathbf{2 4}$ & $\mathbf{3 7}$ & $\mathbf{2 0 0 1}$ \\
Moins d'une fois & 6 & 5 & 5 & 295 \\
\hline Total & 100 & 100 & 100 & 5452 \\
\hline
\end{tabular}

Comme l'indique le tableau 3, une proportion significative d'utilisateurs ne sont pas simplement des utilisateurs habituels mais viennent tous les jours - la tendance est particulièrement vraie pour les garçons, alors que plus de filles que de garçons ne se rendent qu'une fois par semaine au centre de jeunes.

Tableau 4 : Répartition (\%) par niveau de scolarisation et âge (tous centres confondus)

\begin{tabular}{|ccccccc|}
\hline \multirow{2}{*}{$\hat{\text { Agge }}$} & \multicolumn{7}{c|}{ Niveau de scolarisation } \\
\cline { 2 - 7 } & Nul & Primaire & Secondaire & Coranique & Total & Nombre \\
\hline$<10$ ans & 0,6 & 87,0 & $\mathbf{1 , 3}$ & 6,1 & 100 & 231 \\
$10-14$ & 0,9 & 75,0 & $\mathbf{2 2 , 5}$ & 1,6 & 100 & 1541 \\
$15-19$ & 1,2 & 15,1 & $\mathbf{8 1 , 0}$ & 2,7 & 100 & 2299 \\
$20-24$ & 3,2 & 24,3 & $\mathbf{6 8 , 6}$ & 3,8 & 100 & 832 \\
$25-29$ & 9,9 & 26,9 & $\mathbf{5 8 , 0}$ & 5,3 & 100 & 283 \\
$30-34$ & 9,3 & 23,3 & $\mathbf{6 5 , 9}$ & 1,6 & 100 & 129 \\
$\geq 35$ ans & 6,6 & 15,3 & $\mathbf{7 4 , 5}$ & 3,6 & 100 & 137 \\
\hline Total & 2,4 & 37,3 & $\mathbf{5 7 , 5}$ & 2,8 & 100 & 5452 \\
\hline
\end{tabular}

Comme l'indique le tableau 4, plus de la moitié des jeunes rencontrés au cours de l'enquête fréquentaient l'enseignement secondaire, même si 16,8 \% seulement des filles de 15 à 19 avaient jamais fréquenté l'école primaire - ce chiffre tombe à 9,3\% pour les femmes âgées de 20 à 24 ans (selon les données de l'enquête EDS de 1998/1999). 
Tableau 5 : Répartition des personnes et contacts en fonction du sexe et de la zone d'intervention (zone d'intervention du donateur)

\begin{tabular}{|c|c|c|c|}
\hline \multirow{2}{*}{$\begin{array}{c}\text { Echange avec une } \\
\text { personne-ressource ou un/e } \\
\text { ami/e au centre de jeunes } \\
\text { (\% dans la colonne) }\end{array}$} & \multicolumn{2}{|c|}{ Sexe du contact } & \multirow{2}{*}{$\begin{array}{c}\text { Total } \\
\% \\
(\mathrm{~N})\end{array}$} \\
\hline & Garçons & Filles & \\
\hline \multicolumn{4}{|l|}{ Dans la zone d'intervention } \\
\hline Oui & 36 & 60 & 41 \\
\hline Non & 64 & 40 & 59 \\
\hline \multirow[t]{2}{*}{ Total } & 100 & 100 & 100 \\
\hline & (2035) & $(500)$ & $(2535)$ \\
\hline \multicolumn{4}{|l|}{ Hors-zone d'intervention } \\
\hline Oui & 29 & 63 & 34 \\
\hline Non & 71 & 37 & 66 \\
\hline \multirow[t]{2}{*}{ Total } & 100 & 100 & 100 \\
\hline & (4773) & $(807)$ & $(5580)$ \\
\hline
\end{tabular}

Figure 1. Proportion des contacts masculins caractérisés par un échange avec une personne-ressource ou un ami au centre de jeunes, par tranche d'âge

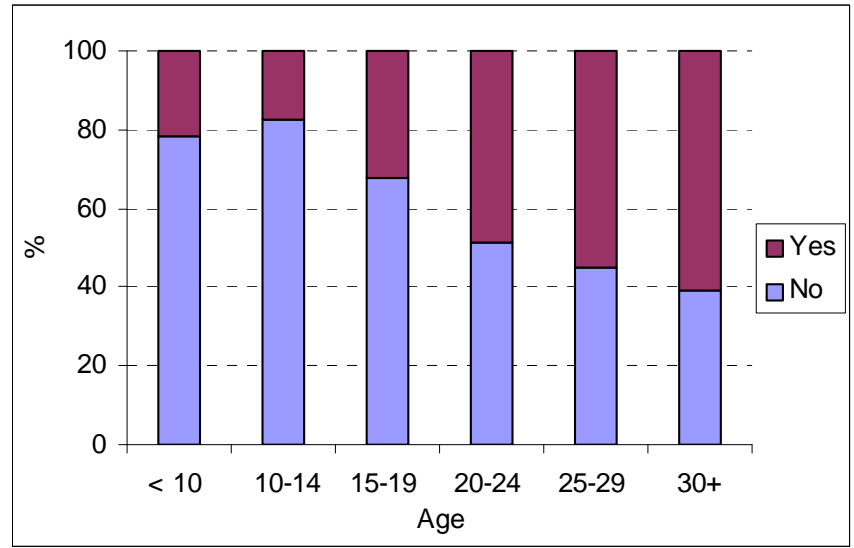


Figure 2. Proportion des contacts féminins caractérisés par un échange avec une personne-ressource ou une amie au centre de jeunes, par tranche d'âge

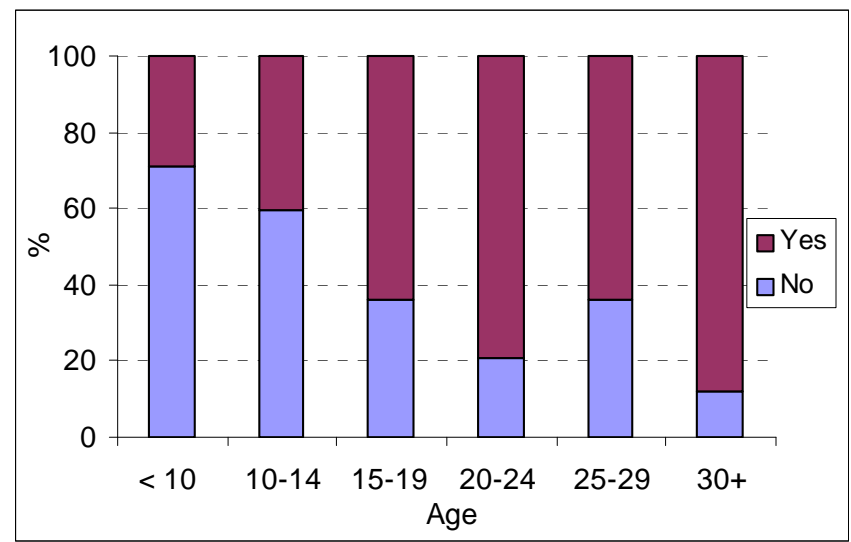




\section{Références}

Bruce, Judith. 2003. «Steps in building evidence-based programs for adolescents » dans «Transitions to Adulthood: Adolescent and Youth Sexual and Reproductive Health: Charting directions for a second generation of programming. » Document de base à l'atelier de l'UNFPA et du Population Council sur la santé sexuelle et reproductive des adolescents et des jeunes, pour la préparation d'une seconde génération de programmes pour adolescents (Adolescent and Youth Sexual and Reproductive Health: Charting Directions for a Second Generation of Adolescent Programming, New York, 1-3 mai 2002).

Enquête démographique et de santé. 1999. «Données sur les adolescents tirées de l'Enquête démographique et de santé -- Tableaux statistiques aux fins de la préparation de programmes: Burkina Faso 1998-1999 ». New York, NY: Population Council.

Mekbib T, Erulkar A, Belete F. 2005. « Who are the targets of youth programs: Results of a capacity building exercise in Ethiopia. » Ethiopian Journal of Health Development, Vol. 19(1): 60 -62. Accessible en ligne (en anglais) à http://www.cih.uib.no/journals/EJHD/eihd19-no1/60.Who\%20are\%20the\%20targets\%20of\%20youth\%20programs.pdf

Population Council et UNFPA. 2005. «Exercice de couverture sur les activités des pairs éducateurs au Burkina Faso. » Voir liste complète des participants dans les remerciements.

Population Council et UNFPA. 2006. « Exercice de couverture sur les activités et la fréquentation des centres de jeunes en Mauritanie. " Voir liste complète des participants dans les remerciements.

Population Council et UNFPA. 2006. «Exercice de couverture sur les activités des pairs éducateurs en Guinée-Bissau. » Voir liste complète des participants dans les remerciements.

«Who is being reached by youth programs? Results from youth-serving organizations' tracking exercise » (Bénéficiaires des programmes de jeunesse : résultats d'un exercice de suivi d'organisations pour les jeunes). 2002. Avant-projet. Population Council, PACT Ethiopia, DSW et Family Guidance Associate of Ethiopia. 